\title{
1 Structural basis of human IL-18 sequestration by the decoy receptor IL- 218 binding protein (IL-18BP) in inflammation and tumor immunity
}

3

4

5

6

\author{
Sammy Detry ${ }^{1,2, \#}$, Julie Andries ${ }^{1,2, \#}$, Yehudi Bloch ${ }^{1,2}$, Cem Gabay $^{3}$, Danielle Clancy ${ }^{1,2}$, Savvas N. Savvides ${ }^{1,2, *}$ \\ ${ }^{1}$ Unit for Structural Biology, Department of Biochemistry and Microbiology, Ghent University, Technologiepark 71, 9052 Ghent, \\ Belgium. \\ 2Unit for Structural Biology, VIB Center for Inflammation Research, Technologiepark 71, 9052 Ghent, Belgium. \\ 3Division of Rheumatology, Department of Medicine, Geneva University Hospitals \& Faculty of Medicine University of Geneva, \\ CH-1211 Geneva 14, Switzerland. \\ \#These authors contributed equally. ; *Corresponding author: savvas.savvides@ugent.be
}

\section{ORCID}

Sammy Detry:

0000-0001-6981-5804

Julie Andries:

0000-0001-8446-9786

Yehudi Bloch:

0000-0001-7924-3539

Cem Gabay:

0000-0001-6853-3063

Danielle Clancy:

0000-0002-8700-3356

Savvas N. Savvides:

0000-0003-3420-5947

\section{Keywords}

Interleukin 18, Interleukin 18 binding protein, crystal structure, decoy receptor, inflammation, cytokine storm, viral infection

\begin{abstract}
Human Interleukin-18 (IL-18) is an omnipresent pro-inflammatory cytokine of the IL-1 family with central roles in autoimmune and inflammatory diseases, and serving as a staple biomarker in the evaluation of inflammation in physiology and disease, including the inflammatory phase in COVID-19. The sequestration of IL-18 by its soluble decoy receptor IL-18 Binding Protein (IL-18BP) is critical to the regulation of IL-18 activity. Since an imbalance in expression and circulating levels of IL-18 is associated with disease, structural insights into how IL-18BP outcompetes binding of IL-18 by its cognate cell-surface receptors would be highly desirable. However, the structure of human IL-18BP in complex with IL-18 had remained elusive. Here, we elucidate the sequestration mechanism of human IL-18 mediated by IL-18BP based on the crystal structure of the IL-18:IL-18BP complex. These detailed structural snapshots reveal the interaction landscape leading to the ultra-high affinity of IL-18BP towards IL-18 and identify substantial differences with respect to previously characterized complexes of IL-18 with IL-18BP of viral origin. Furthermore, our structure captured a fortuitous higher-order assembly between IL-18 and IL-18BP coordinated by a disulfide-bond distal to the binding surface connecting IL-18 and IL-18BP molecule from different complexes, resulting in a novel complex with 2:2 stoichiometry. This tetrapartite assembly was found to restrain IL-18 activity more effectively than the canonical 1:1 complex. Collectively, our findings will provide a framework for innovative structure-driven therapeutic strategies and further functional interrogation of IL-18 in physiology and disease.
\end{abstract}




\section{Significance statement}

Elevated levels of interleukin-18 (IL-18) have long been implicated in numerous inflammatory diseases while also displaying potent anti-tumoral activities. Recent research on COVID-19 has now underscored the role of IL-18 and IL-18 binding protein (IL-18BP), a soluble receptor serving to regulate IL-18 activity, as key players in viral immunity and as promising biomarkers and predictors of disease severity. In this work, we present detailed structural insights into how human IL-18 and IL-18BP interact thereby completing the structural repertoire of IL-18 in complex with its cognate human receptors and viral decoy receptors. Our findings will support structure-based efforts to either disrupt or enhance the interactions of IL-18 with its cognate receptors for therapeutic purposes.

\section{Introduction}

Originally discovered in mice as Interferon- $\psi$-inducing factor (IGIF), Interleukin-18 (IL-18) was found to be a potent inducer of interferon- $\gamma$ (IFN- - ) production in synergy with IL-12 upon exposure to intracellular pathogens (1-3). It was subsequently cloned and identified as an $18 \mathrm{kDa}$ cytokine belonging to the IL-1 cytokine family, and renamed to IL-18 (4). Similar to other IL-1 family cytokines, IL-18 is expressed as an inactive precursor protein that requires cleavage by caspase-1 for full biological activity $(5,6)$. While approximately $80 \%$ of pro-IL-18 is retained intracellularly, the rest is released by macrophages/monocytes and dendritic cells after maturation by caspase-1 $(7,8)$. Mature IL-18 initiates signaling by establishing a tripartite complex with IL-18 receptor alpha (IL-18R $\alpha)$ and IL-18 receptor beta (IL-18Rß), the latter also known as IL-18 receptor accessory protein and IL-1R7. The heterodimerization of the receptors' intracellular Toll/LL-1 receptor (TIR) domains triggers recruitment of the adaptor protein myeloid differentiation primary response 88 (MyD88) which further activates the downstream signaling cascade through IRAK-1/4, TRAF6 and NFKB (9-12). IL-18 is mainly involved in the activation of NK and Thelper 1 (Th1) cells and IFN- - production in response to intracellular bacteria or viruses $(13,14)$.

Due to its highly proinflammatory and pleiotropic activity, IL-18 signaling is highly regulated at many levels to prevent uncontrolled inflammation. Akin to other IL-1 family cytokines, this regulation is achieved through gene regulation (15), caspase-1 activation $(5,6)$ and importantly, is also mediated by a soluble decoy receptor (16). Once IL-18 is activated and released from immune cells, its availability is mainly regulated by sequestration by IL-18 binding protein (IL-18BP), a dedicated soluble decoy receptor-like protein capable of blocking the biological activity of IL-18 (17). Even though IL-18BP functions as a decoy receptor, it is not homologous to any extracellular domain of the IL-18 receptors and is encoded as a separate gene (18), an oddity in the cytokine field (19). However, bioinformatic approaches have proposed that IL-18BP is evolutionary related to TIGIRR-1 (aka IL-1R9) and is thus regarded as a member of the IL-1 receptor family. In addition, sequence and functional homology lead to the identification of IL-18BP orthologs in all orthopoxviruses (20,21), including ectromelia virus (ectvlL-18BP) and Yaba-Like Disease Virus (yldvIL-18BP) $(22,23)$, that act as virulence factors by attenuating immune responses mediated by IL18. 
Not only is human IL-18BP present in the serum at a 20-fold molar excess (24), it also has an exceptionally high affinity ( $\mathrm{K}_{\mathrm{D}} \sim 300 \mathrm{pM}$ ) for IL-18 (25), in contrast to IL-18Ra's affinity towards hIL-18 which is reported to be several orders of magnitude weaker ( $\mathrm{K}_{\mathrm{D}} \sim 69 \mathrm{nM}$ ) (26). Intriguingly, the affinity of viral IL-18BPs for human IL-18 is considerably lower than human IL-18BP ( $\left.\mathrm{K}_{\mathrm{D}} \sim 1 \mathrm{nM}\right)$, although they display picomolar affinity towards mouse IL-18 (25). As a result, IL-18 is sequestered by IL-18BP under homeostatic conditions, thereby preventing it from signaling through its receptors and evoking unwanted pro-inflammatory responses. Interestingly, IL-18-induced IFN- $\gamma$ upregulates IL-18BP expression, creating a negative feedback loop to dampen and resolve inflammation $(27,28)$. Several (auto)immune diseases have been associated with increased levels of IL-18 such as rheumatoid arthritis (RA) (29, 30), Crohn's disease (CD) (31, 32), and systemic lupus erythematosus (SLE) (33-35). As IL-18BP has a high IL-18 sequestration capacity (35), the balance between IL-18/IL-18BP and the concentrations of free IL-18, instead of total IL-18, are more relevant to measure to evaluate inflammatory responses (36). Elevated levels of free IL-18 have been identified in hyperinflammatory diseases such as macrophage activation syndrome (MAS), and systemic juvenile idiopathic arthritis $(28,37)$. Furthermore, in mouse models of MAS, IL-18BP-deficient mice developed more severe disease manifestations, pointing to the crucial role of IL-18BP activity in this setting (28). In addition, elevated levels of IL-18 in blood and bronchoalveolar lavage fluid (BALF) from coronavirus patients have been shown to correlate with COVID-19 disease severity and worse clinical outcomes (3840).

Unsurprisingly, IL-18 neutralizing antibodies or recombinant IL-18BP have been successfully used to mitigate IL-18 related pathologies $(41,42$ ). Clinical trials using recombinant hIL-18BP (Tadekinig $\alpha$ ) for Adult-onset Still's disease (AOSD), rheumatoid arthritis (RA) and plaque psoriasis show promising safety and indicative signs of efficacy in patients with $\operatorname{AOSD}(43,44)$. On the other hand, administration of recombinant IL-18 in mouse tumor models was shown to elicit favorable anti-tumoral effects in synergy with chimeric antigen receptor T (CAR-T) and immune checkpoint inhibitors (45). Whereas such findings provided a strong rationale for the therapeutic potential of IL-18 in cancer (46), its efficacy has not lived up to expectations (47), the culprit being the concomitant increase in the serum concentration of IL-18BP by up to 100 -fold in patients $(46,48)$.

To aid further advancements in the mechanistic interrogation of IL-18 sequestration by IL-18BP and the therapeutic targeting of hIL-18 and hIL-18BP in infectious diseases, inflammation and cancer, structural details of their complex and of interaction interface to high resolution would be opportune. Here, we present the crystal structure of human IL-18 in complex with human IL-18BP. This study shows that hIL18BP binds hIL-18 at the same epitope as IL-18R $\alpha$ and viral IL-18BPs using a large hydrophobic patch flanked by two tightly fitting hydrophobic pockets complemented by salt bridges. A crucial advance in our crystallization efforts was the elimination of the flexible $\mathrm{N}$-terminus and minimization of heterogenous glycosylation patterns of hIL-18BP while retaining functional and biological activity. Moreover, we discovered a novel disulfide-linked interface resulting in an unexpected tetrameric assembly of hIL-18 and hIL-18BP. The structural model presented here is imperative to validate previous models, map key differences between human and viral orthologs of IL-18BP and allow advancements in the design of novel inhibitors or antagonists. 


\section{Results}

\section{Human IL-18BP can be truncated to a bioactive core structure with reduced glycosylation}

To enable structural studies of the human IL-18BP:IL-18 complex by X-ray crystallography we initially considered clinical grade human IL-18BP (Tadekinig alfa) that was used in a phase 2 clinical trial to evaluate the safety and efficacy of IL-18BP in adult-onset Still's disease (43). However, due to the extensive glycosylation of this protein and the need to create glycan-engineered protein amenable to structural studies by X-ray crystallography we produced full-length hIL-18BP in transiently transfected HEK293S MGAT1 -/- cells allowing for N-linked glycosylation as Man ${ }_{5} \mathrm{GlcNAc}_{2}$ glycans (49) and mature hIL-18 in E. coli. Using size-exclusion-chromatography (SEC) and in-line multi-angle laser light scattering (MALLS) we found that full-length hIL-18BP was heavily glycosylated with nearly $50 \%$ of its mass accounted for by glycans (Figure 1A and Supplemental Table 1), consistent with predictions (Figure S1A). Furthermore, the distribution of mass across the SEC-MALLS peak for full-length hIL-18BP, the diffuse electrophoretic mobility of the purified protein in SDS-PAGE (Figure 1A\&B), and large shifts in mass upon treatment with endoglucanases and O-glycosidases suggested heterogeneous glycosylation. Based on our experience with producing secreted glycoproteins with $\mathrm{N}$-linked glycosylation and similar protein families in the HEK293T and HEK293S MGAT1 -/- cell lines for structural biology (50-53), we reasoned that this most likely originates from O-glycans and possible differences in $\mathrm{N}$ - and O-glycosylation site occupancy. Initial crystallization trials using purified hIL-18BPFL:IL-18 complex containing glycan-shaved hIL-18BPFL and displaying apparent stoichiometry of 1:1 as evaluated by SEC-MALLS, did not lead to candidate crystallization conditions even when employing hIL-18BPFL:IL-18 complex up to $30 \mathrm{mg} / \mathrm{mL}$. Such apparent high solubility during crystallization trials was deemed to be consistent with the observed extensive and heterogeneous glycosylation of hIL-18BPFL (Figure $1 \mathrm{~A}$ and Supplemental Table 1). Therefore, we sought to produce alternative versions of hIL-18BP that would be more amenable to structural studies. Sequence alignment of hIL-18BP with homologous viral IL-18BPs, for which structural information is available $(22,23)$, suggested that the expected core beta-sandwich domain of hIL-18BP (residues 63-171) might be flanked by N- and Cterminal stretches (residues 31-62 and 170-194, respectively) bearing intrinsic disorder and the majority of the predicted O-glycosylation sites (Figure S1A and S1B).

To this end we produced hIL-18BP lacking the $\mathrm{N}$ - and/or C-terminal regions in HEK293S MGAT1 $\%$ cells. We found that only hIL-18BP truncated at the N-terminus (residues 63-194), hereafter termed hIL$18 \mathrm{BP}_{\triangle \mathrm{N}}$, could be sufficiently produced for structural studies. SEC-MALLS analysis of hIL-18BP $\mathrm{BN}_{\triangle \mathrm{N}}$ revealed the loss of $3 \mathrm{kDa}$ of protein mass and $5.5 \mathrm{kDa}$ of glycans (Figure $1 \mathrm{~A}$ and Supplemental Table 1), confirming that the N-terminus of hIL-18BP is heavily glycosylated. Additional trimming of N-linked Man ${ }_{5} \mathrm{GlNAc}_{2}$ glycans using Endoglycosidase $\mathrm{H}$ (Endo $\mathrm{H}$ ) resulted in a further loss of $2.5 \mathrm{kDa}$ of $\mathrm{N}$-linked glycans (Figure $1 \mathrm{~A}, 1 \mathrm{~B})$.

We initially verified the functional activity of hIL-18BP ${ }_{\triangle N}$ treated with Endo $\mathrm{H}\left(\mathrm{IL}-18 \mathrm{BP} \mathrm{P}_{\triangle \mathrm{N}-\mathrm{EH}}\right)$ in terms of its ability to sequester hIL-18 in stoichiometric fashion and high affinity. Indeed, SEC-MALLS analysis showed that hIL-18BP $\triangle$ N-EH can establish a monodisperse complex with hIL-18 according to a 1:1 
stoichiometry (Figure $1 \mathrm{C}$ and Supplemental Table 1). Using isothermal titration calorimetry (ITC), we compared the thermodynamic binding profiles and affinities of hIL-18BP FL, hIL-18BP $\triangle N$-EH, and the hIL-18 cognate receptor (IL-18R $\alpha$ ) to hIL-18 (Figure 1D). Our data show that hIL-18BP $\triangle \mathrm{N}-\mathrm{EH}$ and hIL-18BPFL are virtually indistinguishable from each other in their thermodynamic binding profiles and sub-nM affinities towards hIL-18. In contrast and consistent with previously published data (26), human hIL-18R $\alpha$ binds to hIL-18 with lower affinity $\left(K_{D}=4.7 \mathrm{nM}\right)$ than hIL-18BP. However, the binary complex affinity measured here is markedly higher than previously reported affinities $\left(\mathrm{K}_{\mathrm{D}} \sim 60 \mathrm{nM}\right)(26)$ by surface plasmon resonance.

Finally, we interrogated the biological activity of hIL-18BP $\mathrm{PN}_{\triangle \mathrm{EH}}$ by using the hIL-18-responsive macrophage cell line, KG-1, the activity of which is manifested by NFKB signaling and pro-inflammatory cytokine and chemokine production (54). In this context, we measured the amount IL-8 as secreted from KG-1 cells upon hIL-18 stimulation, and found that both hIL-18BP $F$ and hIL-18BP ${ }_{\triangle N-E H}$ displays comparable inhibitory activities. (Figure 1E).

\section{Structural mimicry underlies the sequestration of human IL-18 by IL-18BP}

Structural insights into the sequestration of human IL-18 by its cognate decoy receptor IL-18BP had remained elusive despite the growing importance of IL-18BP in physiology and disease. Using the truncated and glycan-shaved hIL-18BP $P_{\triangle N-E H}$ we purified crystallization-grade hIL-18: hIL-18BP $P_{\triangle N-E H}$ complex and determined the crystal structure of the complex to $1.8 \AA$ resolution by molecular replacement using crystal structure of ectromelia poxvirus IL-18 binding protein (ectvIL-18BP) (23) (pdb 3f62) as a search model (Figure 2A, Table 1). Crystallographic refinement and the quality of the ensuing electron density maps were enhanced after correcting the X-ray data for data anisotropy using the STARANISO server (55). The crystal asymmetric unit contains one copy of the 1:1 complex and reveals how hIL-18BP uses the side of its h-type immunoglobulin $\beta$-sandwich fold to bind the $\beta$-trefoil structure hIL-18 (Figure 2A). hIL-18BP harbors two internal disulfide bridges connecting strands $A$ and $B$ (Cys86-Cys150), and B and F (Cys64-Cys89). In addition, three predicted $\mathrm{N}$-glycan sites are occupied by an $\mathrm{N}$-Acetylglucosamine residue (GlcNAc) at residues Asn79, Asn103 and Asn147. Our model for hIL-18BP starts at the beginning of the hIL-18BP ${ }_{\Delta N}$ construct, i.e. Gln63, however no density was observed past Ala170 in the crystal solvent channels, consistent with the predicted disorder of the C-terminal tail (Figure 2C, Figure S1B). Human IL-18 features its signature $\beta$-trefoil fold and closely resembles the structure of unbound hIL-18 (pdb 3wo2) (all-atom r.m.s.d.=1.8 $\AA$ ). Structure-based sequence alignments of human IL-18BP and structural superpositions against the two structurally characterized viral IL-18BPs, ectromelia virus IL-18BP (ectvIL-18BP) and yabalike disease virus disease IL-18BP (yldvIL-18BP), and domain 3 of hIL-18R $\alpha$ (hIL-18R $\alpha_{\mathrm{D} 3}$ ) establish the strong conservation of the adopted fold, albeit with major variations in the CD loop mediating hIL-18 binding and AB loop (Figure 2B, Figure 2C, Figure 2D). Importantly, these analyses reveal that structural mimicry and direct steric competition underlie the sequestration of human hIL-18 by hIL-18BP (Figure 2C).

Specifically, human IL-18BP covers the top of the hIL-18 $\beta$-barrel with one side of its $\beta$-sandwich scaffold composed of strands $C, G$ and $F$ and is slightly inclined to enable protrusion of loops $F G$ and $B C$ into the barrel. Additionally, residues from the CD loop and $\mathrm{D}$ strand mediate important interactions on the other 
side of the sheet (Figure 2A). In light of the plethora of structural information on human/viral IL-18BPs and human IL-18R $\alpha$ in complex with hIL-18, curation and comparison of the observed interaction interfaces to distil key structure-function insights presents with considerable challenges. At the same time, annotation of amino acid positions that have been interrogated by mutagenesis would also be desirable. Together, such analyses could lead to a rationale for the exquisitely efficient sequestration of hIL-18 by hIL-18BP and might further fuel protein engineering approaches to modulate this sequestration potential. Therefore, we sought to develop a representation scheme that would allow effective parallel comparison of all structural data available together with mutagenesis data (Figure 3B). The binding interface can be subdivided into three sites: Site A consists of complementary hydrophobic patches on both hIL-18 and hIL-18BP. Sites B and $\mathrm{C}$ each engage a phenylalanine on hIL-18BP, Phe106 and Phe95 respectively, to protrude into a hydrophobic cavity on hIL-18 sealed by salt bridges and hydrogen-binding interactions (Figure 3A). Sequence alignments of hIL-18BP orthologs among vertebrates (Figure S2A) allows mapping of highly conserved residues on the surface of hIL-18BP that mediate sequestration of hIL-18, and shows that this region of hIL-18BP is the most conserved compared to any other part of the decoy receptor (Figure S2B). This suggests that the observed human IL-18BP:IL-18 complex can serve as a structural representative for all such complexes across species. When comparing the interface of human IL-18BP with its viral counterparts, these phenylalanine residues are largely conserved, with the exception of yldvIL-18BP, which contains a threonine instead of phenylalanine at site $B$. The most striking difference is the lack of salt bridges in viral IL-18BPs at site C. Conversely, human IL-18R $\alpha$ does form these salt bridges but lacks the phenylalanine residues protruding into the hydrophobic cavity of hIL-18, while also lacking another hydrophobic substitute. At site B, hIL-18R $\alpha$ also lacks the phenylalanine residue, but substitutes it for a methionine to fill the hydrophobic pocket (Figure 3B). Overall, site A is very similar for all interfaces, with mainly conservative substitutions of key interacting residues. The absence of these phenylalanine residues may explain the higher affinity of human IL-18BP to IL-18 than its receptor. Similar to IL-18Ra, but in contrast to the viral IL-18BPs, human IL-18BP exploits additional interactions via two side-chain-to-mainchain hydrogen bonds at site $A$.

\section{A disulfide-linked interaction site mediates a novel higher-order IL-18:IL-18BP complex}

An intriguing feature of the IL-18-IL-18BP complex concerns an unexpected disulfide bridge between Cys74 of hIL-18 in the canonical IL-18:IL-18BP complex and Cys131 of IL-18BP participating in a symmetry-related complex. This results in a covalent tetrameric assembly with a 2:2 stoichiometry and twofold symmetry (Figure 4A). We note that such higher-order assembly was not observed in the preparatory stages of precrystallization samples as assessed by SEC-MALLS or SDS-PAGE (Figure 1B and 1C). However, the sample was concentrated to $30 \mathrm{mg} / \mathrm{ml}$ prior to crystallization. To verify whether such a higher order complex originated during this final protein concentration step, the concentrated sample was diluted back to 0.5 $\mathrm{mg} / \mathrm{ml}$ and analyzed by SDS-PAGE and SEC-MALLS. SDS-PAGE shows a band at $~ 38 \mathrm{kDa}$ which can be resolved to the constituent protein components upon the addition of the reducing agent DTT prior to loading the sample, indicating the presence of a disulfide linked dimer (Figure 4B). We confirmed by mass 
spectrometry that both hIL-18 and hIL-18BP were present in this disulfide linked protein adduct, verifying a heterodimer as opposed to a homodimer of either. Additionally, SEC-MALLS shows that more than $90 \%$ of the previously concentrated sample now exists as a tetramer of $74 \mathrm{kDa}$, rather than the expected canonical IL-18:IL-18BP complex of $37 \mathrm{kDa}$ (Figure 4C). Protein conjugate analysis of SEC-MALLS data validated the presence of both hIL-18BP and hIL-18 in the complex with a 2:2 stoichiometry, as any other combination of proteins and their glycans would not be compatible with their respective molecular masses.

Interestingly, in the structure of unbound hIL-18 and when in complex with its decoy and signaling receptors, the cysteine involved in the observed novel disulfide bond with IL-18BP is part of a $3_{10}$ helix and is not surface exposed (Figure 4A, left panel). This region in inbound IL-18 is rather amphipathic in terms of physicochemical properties and interacts with several detergent molecules (CHAPS) in pdb 3wo2 (26) . Thus, to participate in the observed disulfide bond, the cysteine has flipped outwards and is accompanied by a structural rearrangement thereby disrupting the helical structure (Figure 4A, left panel). Interestingly, an ensemble of structural models of IL-18 determined by NMR has shown this helix to be very flexible (56), which would favor such dynamics. We note that for the reported crystals structure of hIL-18 complexed with ectvIL-18BP and yldvIL-18BP (pdb $3 f 62$ and 4eee respectively), all free cysteines were mutated to serine, thereby excluding the possibility of such a disulfide linkage, although this particular helix/loop segment was not modelled due to its apparent flexibility. Surrounding the cysteine disulfide bridge is a novel interface mediated by the beta strands E, B, D and D' of the opposite side of the beta-sandwich compared to the canonical interface and is maintained by a several hydrophobic residues on both 251 molecules.

Because this hetero-tetramer was only initially observed with IL-18BP $\triangle \mathrm{N}$-EH lacking the N-terminal and Nglycans, we also investigated if a similar higher-order complex could be formed with IL-18BPFL or

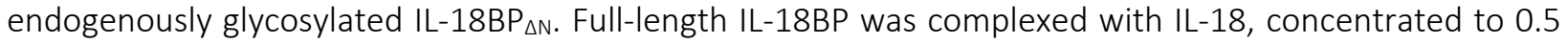
$\mathrm{mg} / \mathrm{mL}$ and analyzed by SEC-MALLS. No tetrameric complex was observed using IL-18BP FL, either in its glycosylated or deglycosylated form, suggesting that removal of the $\mathrm{N}$-terminal segment of IL-18BP promotes the formation of this hetero-tetrameric complex (Figure $4 C$ ). We note that Cys51 is part of the truncated and likely pairs up with Cys131 in the full-length protein, thus preventing heterotetramer formation. Endogenously glycosylated IL-18BP $\mathrm{PN}_{\Delta \mathrm{N}}$, without Endo $\mathrm{H}$ treatment, also formed a tetrameric complex with IL-18 at high concentrations, albeit at a lower rate than IL-18BP ${ }_{\triangle N-E H}$ (Figure 4D).

The presence of a disulfide bridge linking IL-18BP $\mathrm{BN}_{\Delta \mathrm{N}}$ and IL-18 distal to the binding interface in the hetero-tetrameric assembly observed here may result in better sequestration and inhibition of IL-18 than in the canonical 1:1 complex. Furthermore, the site where disulfide-linked IL-18BP engages on IL-18 would

264 also sterically clash with the eventual binding site of IL-18 to domain 1 of IL-18R $\alpha$. To test this, both the 265 heterodimeric or hetero-tetrameric complex of IL-18BP $\mathrm{BN}_{\triangle \mathrm{EH}}$ and IL-18 was purified and used to stimulate 266 KG-1 cells. While both the dimeric and tetrameric complex inhibited IL-18 activity compared to IL-18 alone, 267 the tetrameric assembly restrained IL-18 activity more effectively than the dimeric complex, resulting in 268 less inflammatory cytokine production from KG-1 cells (Figure 4E). 


\section{Discussion}

271 Over the past two decades and in the absence of an experimentally determined structure for the human

272 IL-18-IL-18BP complex, numerous studies have used orthogonal methods to predict how human IL-18BP

273 interacts with human IL-18 to efficiently sequester and prevent signaling. To date, structural features and

274 binding interfaces between human IL-18 and human IL-18BP have been inferred from computational

275 models (44), human and viral mutagenesis studies (45-49) and homologous structures of viral IL-18BPs in

276 complex with human IL-18 $(22,23)$. Additionally, the ternary assembly of human IL-18 in complex with its

277 cognate receptors, IL-18R $\alpha$ and IL-18R 3 (26), has shed some light on the potential for competitive binding

278 of human IL-18BP. The high-resolution structural analysis of human IL-18BP bound to IL-18 presented here

279 is imperative to further understand and exploit the ultra-high affinity binding mode that characterizes this

280 unique decoy receptor-like protein $(22,23)$. Human IL-18BP binds the same epitope of hIL-18 used by viral

281 IL-18BPs to compete with the third ectodomain of hIL-18R $\alpha$. The majority of the interface (Site A) consists

282 of two large, complementary hydrophobic patches, mimicking the landscape of hydrophobic peaks and

283 troughs on each side of the interface, supplemented with two side-chain-to-main-chain hydrogen bonds.

284 Comparing the structure of hIL-18BP with its viral orthologs confirms that this large hydrophobic patch on site $A$ is highly conserved. yldvIL-18BP contains some additional hydrophobic residues (Ile78 and Leu80) on the DE loop interacting with hIL-18 (Leu 5 and Glu6) at the perimeter of the interface. In all other structures, except yldvIL-18BP, this loop is constrained as an additional $\beta D^{\prime}$ strand. On each side of the hydrophobic patch, a phenylalanine on hIL-18BP is tightly inserted inside hydrophobic cavities on hIL-18 and additionally locked down by salt bridges (Site B and C, Figure 3B). Mutating either phenylalanine residues to alanine reduces the ultra-high picomolar affinity of hIL-18BP down to low-nanomolar affinity $(22,23,57)$. Mutations of the corresponding residues in viral IL-18BPs have a similar effect, highlighting the importance of these specific residues for binding. Interestingly, in hIL-18R $\alpha$ the corresponding residue is either substituted by methionine (Site B) or completely lacking (Site C), although site B is flanked by additional hydrophobic residues residing on hIL18Ra's longer CD' loop. The absence of these seemingly critical phenylalanine residues may, in part, explain the higher affinity of hIL-18BP to IL-18 than its receptor. Similarly, mutating Glu108 and Glu106 on hIL-18BP involved in the salt bridges at Site B accordingly resulted in a lower affinity, as well as mutating Lys53 on hIL-18 which is located on the other end of the salt bridges $(58,59)$. Mutations in Site $C$ particularly have not been reported, because in both viral structures the corresponding residues (Glu48 in ectvIL-18BP and His51 in yldvIL-18BP) are not involved in salt bridge formation. Overall, it is clear that hIL-18BP utilizes a unique constellation of key residues, contributing to ultra-high affinity binding to IL-18, however further investigation is required to determine binding hotspots important for this interaction. Whether or not the lower affinity of viral IL-18BPs is actually advantageous or disadvantageous for their function is unclear.

Unexpectedly, we observed a higher order assembly of IL-18 and IL-18BP in our crystallographic 

canonical dimeric 1:1 complex. Given that high concentrations and removal of the N-terminal region appear to be required, it is likely not biologically relevant. However, this fortuitous observation suggests that this interface may be amenable to further engineering to increase the sequestration potency of IL-18BP, possibly in combination with domains 1 and 2 of IL-18R $\alpha$. It brings to light plasticity in IL-18, the possible reactivity of Cys131 in IL-18BP, and the possible utilization of a hydrophobic patch at opposite ends to the IL-18:IL-18BP interface to create a new interaction interface. An analogous yet distinct bivalence has been observed in viral yldvIL-18BP. This ortholog lacks a highly conserved phenylalanine residue in site B, however it compensates for this by forming a 2:2 complex with IL-18, driven by disulfide-linked IL-18BP homodimerization (23). However, it is important to note that the 2:2 hetero-tetrameric complex seen here for human IL-18BP engages a separate interface, and is driven by an additional interaction with IL-18 319 cytokine.

The structural model presented here will facilitate further the structure-driven development of novel therapeutics to either disrupt or mimic hIL-18BP binding. Aberrant IL-18 signaling has been implicated in numerous inflammatory diseases including RA, AOSD, SLE, sickle cell disease, and more recently, the inflammatory phase of coronavirus infection. $(28,29,36,37,60)$. Coronavirus disease (COVID-19), caused by the SARS-CoV2 virus, displays a broad range of clinical symptoms, resulting in disease severity extending from mild to fatal outcomes. Accumulating evidence has shown that tissue damage in the later stages of severe COVID-19 infection is driven by cytokine release syndrome (CRS), also known as cytokine storm (38, $39,61)$. Recent studies have detected elevated levels of IL-18 in patients with moderate and severe COVID19 infection, which strongly correlated with disease severity $(38,39,62)$. Longitudinal analysis of patients infected with SARS-CoV-2 showed IL-18 levels remained elevated in severe COVID-19 patients admitted to ICU, while cytokine levels declined over time in moderately affected patients. Moreover, both IL-18 and IL18BP have been identified as promising biomarkers to predict disease severity and likelihood of death after SARS-CoV-2 infection $(38,63)$. Elevated levels of IL-18 were also detected in multisystem inflammatory syndrome (MIS) and Kawasaki syndrome in children following SARS-CoV-2 infection, and may be used as a biomarker to distinguish these diseases from similar hyperinflammatory syndromes (37). Increased IL-18 production has also been associated with activation of cytotoxic mucosal-associated invariant T (MAIT) cells during coronavirus infection, leading to lung tissue damage and increased disease severity (40). These studies suggest that modulation of IL-18 activity may represent a novel therapeutic opportunity for COVID19, however, no clinical trials with recombinant IL-18BP or IL-18-specific antagonistic have yet been initiated $(64,65)$. However, because of the pleiotropic activity of IL-18, there will likely be a narrow therapeutic window to exploit the beneficial activity of IL-18 for effective viral clearance, while avoiding aberrant inflammation and tissue damage associated with high IL-18 activity during late-stage infection. In addition, IL-18 has been shown to display potent anti-tumor activity, however clinical trials using recombinant IL-18 have shown limited efficacy (42). IL-18BP, produced in the tumor microenvironment, has been identified as an 'immune checkpoint', hampering the therapeutic application of recombinant IL18 in cancer (66). Directed evolution of yeast-displayed mouse IL-18 variants identified a decoy-resistant

346 IL-18 that retains signaling activity but escapes inhibition by IL-18BP. This IL-18 variant displayed potent cytotoxic $T$ cell activation, NK cell maturation and tumor growth inhibition compared to its wildtype 
counterpart (66). The high-resolution structural models presented here, in particular the experimentally determined interaction interface of IL-18 and human IL-18BP, will be of crucial importance for additional protein engineering and future drug design for the development of novel therapeutics for use in viral infections, autoimmune and inflammatory diseases and cancer.

\section{Materials and Methods}

\section{Plasmids, protein expression constructs and cell Lines}

All constructs were created by a traditional restriction ligation approach. Restriction enzymes, T4 ligase and Q5 polymerase were purchased from NEB (New England Biolabs, Ipswich, Massachusetts, USA). All primers were purchased from IDT (Integrated DNA Technologies, Leuven, Belgium). Recombinant DNA was purchased from GenScript (GenScript, Piscataway, New Jersey, USA). The mature sequence of human IL-18 (residues 37-193, UniProt ID: Q14116) was codon optimized for expression in E. coli and purchased in the pUC57 vector from GenScript (GenScript, Piscataway, New Jersey, USA). The sequence was cloned into the pET42a plasmid (Cat No 70561, Novagen, Merck, Overijse, Belgium) in frame with an N-terminal caspase3cleavable glutathione S-transferase (GST)-tag (26). The introduction of an extra N-terminal hexahistidine (His 6 )-tag was performed by polymerase chain reaction (PCR). The sequence of human IL-18BP (residues 1194, UniProt ID: 095998) was purchased in the pUC57 vector at GenScript (GenScript, Piscataway, New Jersey, USA). The sequence was cloned in frame with a C-terminal caspase3 site followed by an AviTag and a His ${ }_{6}$ tag. The construct was further cloned in frame with an N-terminal chicken RTP $\mu$-like signal peptide sequence (67) that replaced the native signal peptide sequence (residues 1-30). For crystallization purposes, the N-terminus (residues 31-60) was removed by PCR (IL-18BP $\mathrm{BN}_{\Delta N}$ ) and the construct was cloned in the same pHLSec plasmid containing an N-terminal chicken RTP $\mu$-like signal peptide sequence and a caspase 3 cleavable Avi-His6-tag. All constructs were validated by Sanger sequencing by GATC BioTech (Konstanz, Germany) before further experiments were performed.

\section{Expression and purification of recombinant Proteins}

BL21(DE3) E. coli transformed with plasmid pET42 expressing N-terminally GST- and HIS-tagged human IL-18 were grown at $37^{\circ} \mathrm{C}$ in Lysogeny broth (LB) medium containing kanamycin $(25 \mu \mathrm{g} / \mathrm{ml})$ as selection marker for the pET42a plasmid (Cat No 70561, Novagen, Merck, Overijse, Belgium). When the optical density at $600 \mathrm{~nm}$ (OD600) reached 0.6, the expression of human IL-18 was induced by addition of isopropyl- $\beta$-D-thiogalactopyranoside (ITPG) at a final concentration of $1 \mathrm{mM}$, after which the culture was 
incubated at $28^{\circ} \mathrm{C}$ for 5 hours. The bacteria were harvested by centrifugation $\left(7,000 \times \mathrm{g}\right.$ for $10 \mathrm{~min}$ at $\left.4^{\circ} \mathrm{C}\right)$ and the cellular pellet was stored at $-80^{\circ} \mathrm{C}$. The bacterial pellet was thawed and resuspended in HEPES buffered saline (HBS, 20 mM HEPES, 150 mM NaCl, pH 7.4) with DNasel (REF 10104159001, Roche Diagnostics, Vilvoorde, Belgium). The cells were lysed by sonication with a Qsonica macrotip sonicator (Newtown, Connecticut, USA) (on-time 4 min; pulse on $30 \mathrm{sec}$; pulse off $30 \mathrm{sec}$; amplitude 70\%) while cooled on ice. The lysate was clarified by centrifugation $\left(20,000 \times \mathrm{g}\right.$ for $45 \mathrm{~min}$ at $\left.4^{\circ} \mathrm{C}\right)$ and filtration using a 0.22- $\mu \mathrm{m}$ Millipore ${ }^{\mathrm{TM}}$ Steritop ${ }^{\mathrm{TM}}$ Sterile Vacuum Bottle-Top Filter (Thermo Fisher Scientific, Merelbeke, Belgium), and loaded onto a HisTrap ${ }^{\mathrm{TM}}$ HP $5 \mathrm{ml}$ column (GE Healthcare, Diegem, Belgium) equilibrated with HBS. The column was washed (HBS, $50 \mathrm{mM}$ imidazole) before eluting the protein (HBS, $250 \mathrm{mM}$ imidazole) following desalting using a HiPrep ${ }^{\mathrm{TM}}$ 26/10 Desalting column (GE Healthcare, Diegem, Belgium) to remove imidazole. The His ${ }_{6}$-GST-tag was removed by caspase 3 (produced in-house) cleavage at $37^{\circ} \mathrm{C}$ for 1 hour. To remove the uncleaved protein and the $\mathrm{His}_{6}{ }^{-G S T}$-tag, the sample was again loaded onto a HisTrap ${ }^{\text {TM }}$ HP 5 ml column (GE Healthcare, Diegem, Belgium). The flow-through was collected and concentrated before injection onto a HiLoad ${ }^{\circledR}$ 16/600 Superdex ${ }^{\circledR} 75$ pg column (GE Healthcare Diegem, Belgium) using HBS as running buffer. Fractions containing human IL-18 were pooled, flash frozen and stored at $-80^{\circ} \mathrm{C}$. The purity of the protein was evaluated on SDS-PAGE stained with Coomassie blue.

\section{Expression and purification of recombinant human IL-18BP}

Adherent HEK293S MGAT1-/- cells (68) were grown in 5-layer cell culture flasks (Falcon ${ }^{\circledR}$ Multi-Flask, Corning, New York, USA) in Dulbecco's Modified Eagle Medium (DMEM) (Gibco' ${ }^{\mathrm{TM}}$, Life Technologies, Thermo Fisher Scientific, Merelbeke, Belgium) supplemented with 10\% Fetal Calf Serum (FCS) (Bodinco, Alkmaar, Netherlands). Upon transfection, the growth medium was exchanged for DMEM supplemented with 3.6 mM valproic acid (item 13033, Cayman Chemical Company Europe, Tallinn, Estonia). Transient expression of human IL-18BP or IL-18BP $\triangle N \Delta N$ was achieved using branched polyethyleneimine (PEI) (Mn 10 000, Cat.:40,872-7, Sigma-Aldrich, Diegem, Belgium) as transfection reagent (67). After 4 days of expression, filtered conditioned medium was loaded onto a $5 \mathrm{ml}$ cOmplete ${ }^{\mathrm{TM}}$ His-tag Purification Column (Roche Diagnostics, Vilvoorde, Belgium). The protein was eluted with $250 \mathrm{mM}$ imidazole in HBS after which the imidazole was removed using a HiPrep ${ }^{\text {TM }}$ 26/10 Desalting column (GE Healthcare, Diegem, Belgium). The Avi-His6-tag was cleaved by caspase-3 (produced in-house) and the glycans were trimmed by EndoH 
407 (produced in-house) at $37^{\circ} \mathrm{C}$ for 1 hour. The flow-through of the HisTrap ${ }^{\mathrm{TM}} \mathrm{HP} 5 \mathrm{ml}$ column (GE Healthcare,

408 Diegem, Belgium) containing the cleaved protein was collected, concentrated and injected onto a HiLoad ${ }^{\circledR}$ 409 16/600 Superdex ${ }^{\circledR} 200$ pg column (GE Healthcare Life Sciences) using HBS as running buffer. Fractions 410 containing human IL-18BP or IL-18BP ${ }_{\Delta N \Delta N}$ were pooled, flash frozen and stored at $-80^{\circ} \mathrm{C}$. The purity of the 411 protein was evaluated on SDS-PAGE stained with Coomassie.

412 Multi-Angle Laser Light Scattering

413 Purified protein samples at a concentration of $0.5 \mathrm{mg} \mathrm{ml}^{-1}$ were injected onto a Superdex Increase 414 10/300 GL column (GE Healthcare, Diegem, Belgium) that was pre-equilibrated with HBS. The column was 415 coupled in line with a UV-detector (Shimadzu, Brussels, Belgium), a miniDAWN TREOS multi-angle laser 416 light scattering (MALLS) detector (Wyatt, Santa Barbara, California, USA) and an Optilab T-rEX 417 refractometer (Wyatt, Santa Barbara, California, USA). Refractive index (RI) increment values (dn/dc) of $4180.185 \mathrm{ml} \mathrm{g}^{-1}$ and $0.155 \mathrm{ml} \mathrm{g}^{-1}$ were used for protein and glycan analysis, respectively. Bovine serum albumin 419 (BSA) (Pierce, Merelbeke, Belgium) was used as standard to correct for band broadening. The resulting data was analyzed using the ASTRA6.1 software (Wyatt, v6.1) and errors were calculated in Microsoft Excel.

\section{Isothermal Titration Calorimetry}

All proteins were buffer matched in HBS from the same batch using size exclusion chromatography and all the solutions were degassed. Protein concentrations were determined with the NanoDrop ${ }^{\mathrm{TM}} 2000$ 424 (Thermo Fisher Scientific, Merelbeke, Belgium) using their corresponding extinction coefficients 425 (absorbance of 1\%). Experiments were carried out on the MicroCal VP-ITC calorimeter (Malvern Panalytical, 426 Malvern, United Kingdom) at $37^{\circ} \mathrm{C}$. Titrations were always preceded by an initial injection of $3 \mu \mathrm{l}$, and were 427 carried out using $10 \mu \mathrm{l}$ injections applied $300 \mathrm{~s}$ apart. The sample was stirred at a speed of 300 r.p.m. 428 throughout. The data was recorded using the Origin ${ }^{\circledR}$ scientific plotting software (Version 7.0, MicroCal, 429 Malvern Panalytical, Malvern, United Kingdom) and further analyzed using NITPIC (69) and SEDPHAT (70) 430 fitting the data with a "one to one" binding model.

\section{Bioactivity assays}

KG-1 cells were cultured in RPMI media (Gibco) supplemented with $10 \%$ fetal calf serum (Bodinco).

433 Cells were cultured at $37^{\circ} \mathrm{C}$ in a humidified atmosphere with $5 \% \mathrm{CO}_{2}$. KG-1 cells were plated at $2 \times 10^{5}$ cells 434 per well in 24-well plates. Recombinant IL-18 was pre-incubated with IL-18BP FL,IL-18BP $\triangle \mathrm{N}$-EH or complexes thereof as indicated, for $1 \mathrm{~h}$ at $37^{\circ} \mathrm{C}$. KG-1 cells were left untreated or stimulated with IL-18 (0.5 nM final 
concentration) +/- IL-18BP, as indicated. Cells were incubated with stimuli for $18 \mathrm{~h}$ and cell culture

437 supernatants were subsequently collected. Culture supernatants were centrifuged for 5 minutes at $1000 \mathrm{~g}$ to remove cells and frozen at $-80^{\circ} \mathrm{C}$.

\section{Detection of cytokines by ELISA}

Human IL-8 was measured from cell culture supernatants using commercially available specific paired ELISA kits (R\&D Systems). Briefly, a 96 well plate was coated with $50 \mathrm{ml}$ of capture antibody per well

442 according to manufacturer's instructions, and incubated overnight at room temperature. Wells were 443 washed three times with $150 \mathrm{ml}$ wash buffer (0.05\% Tween-20 in PBS, pH 7.2), then blocked for $1 \mathrm{~h}$ in 444 reagent diluent (1\% BSA in PBS, pH 7.2). Samples were diluted as required in reagent diluent and $50 \mathrm{ml}$ of each sample was added to wells and incubated for $2 \mathrm{~h}$ at room temperature. Appropriate standards were

446 prepared for each ELISA assay according to manufacturer's instructions. Wells were washed again three

447 times, followed by $50 \mathrm{ml}$ of biotin-conjugated detection antibody incubated for $2 \mathrm{~h}$. Wells were washed 448 three times and $50 \mathrm{ml}$ streptavidin-HRP was added to wells and incubated for 30 minutes in the dark. 449 Finally, wells were washed three times, followed by addition of $50 \mathrm{ml}$ TMB substrate solution. The reaction 450 was stopped with $30 \mathrm{ml}$ of $2 \mathrm{M}$ sulfuric acid and absorbance was read at $450 \mathrm{~nm}$ on an ELISA plate reader 451 (Fluostar). All cytokine assays were carried out using triplicate samples from each culture.

\section{Bioinformatic analyses}

453 All sequence alignments were performed by Clustal Omega (71). Structural alignments were performed in 454 Swiss-PdbViewer (72). Glycan prediction were performed using NetNGlyc (73) and NetOGlyc servers (74). 455 Protein disorder was predicted using IUPred (75). Sequence alignments were visualized by ESPript (76). 456 Protein structures were visualized in $\operatorname{PyMOL}(77,78)$. Interaction interface was inferred using the PISA 457 server (79).

459 During the purification of IL-18BP ${ }_{\Delta N}$, purified IL-18 was added in a 1:2 ratio prior to caspase 3 and Endo $460 \mathrm{H}$ digests. The complex was purified via SEC and concentrated to $35 \mathrm{mgml}^{-1}$. Sitting-drop vapor diffusion 461 experiments were set up in Swissci 96-well triple drop plates (Molecular dimensions, Suffolk, United 462 Kingdom) with commercial sparse matrix crystallization screens. Using a Mosquito ${ }^{\circledR}$ liquid handling robot 463 (TTP Labtech, Hertfordshire, United Kingdom), the protein was mixed with mother liquor in a 1:1 ratio in a 464 final volume of $200 \mathrm{nl}$. The plates were incubated at $20^{\circ} \mathrm{C}$. An initial hit in the Crystal Screen (Cat No HR2- 
466 M Sodium cacodylate trihydrate $\mathrm{pH}$ 6.5, 18\% w/v Polyethylene glycol 8000) was optimized by varying $\mathrm{pH}$

467 (6.2-7.1) and PEG concentration (12\%-27\%). Crystals obtained from the optimization screen were used for seeding new plates, finally yielding single crystals. These crystals were cryoprotected in mother liquor supplemented with $20 \%$ glucose prior to cryo-cooling in liquid nitrogen. Diffraction data was initially collected under cryogenic conditions at a synchrotron radiation source, namely beamline PETRA III in

471 Hamburg.

The diffraction data were integrated using XDS (80) and were treated for anisotropy using the STARANISO server (55). Initial phases were determined by maximum-likelihood molecular replacement in PHASER (81) using a search model derived from the structure of hIL-18 in complex with ectvhlL-18BP (pdb 3f62). Iterative cycles of structure building and refinement were performed in COOT (82) and PHENIX.refine (83) respectively.

477 Structure coordinates and structure factors for the human IL-18:IL18BP complex have been deposited in the Protein Data Bank (PDB) under accession code 7al7.

Acknowledgements

480 We thank AB2 Bio Ltd. (Lausanne, Switzerland) for clinical grade recombinant IL-18BP and Prof. Seamus 481 Martin, Trinity College (Dublin, Ireland), for providing KG-1 cells. J.A., Y.B. and D.C. are research fellows of 482 Research Foundation Flanders (FWO), Belgium. This project has received funding from the European Union's Horizon 2020 research and innovation program under grant agreement No 779295.

\section{Author Contributions}

S.D. and J.A. carried out recombinant protein production and purification, biophysical studies, and structural studies with contributions from S.N.S. D.C. carried out bioactivity assays. Y.B. provided input on the generation of the glyco-engineered hIL-18BP variant. A.S., E.J.S., and C.G. provided initial research materials. S.D., J.A., D.C. and S.N.S. wrote the manuscript with contributions from all authors. S.N.S. conceived and supervised the project.

\section{References}

1. H. Okamura, K. Kawaguchi, K. Shoji, Y. Kawade, High-level induction of gamma interferon with various mitogens in mice pretreated with Propionibacterium acnes. Infect Immun 38, 440-443 (1982).

2. M. Wada, H. Okamura, K. Nagata, T. Shimoyama, Y. Kawade, Cellular mechanisms in in vivo production of gamma interferon induced by lipopolysaccharide in mice infected with Mycobacterium bovis BCG. J. Interferon Res. 5, 431-443 (1985). 
3. K. Nakamura, H. Okamura, M. Wada, K. Nagata, T. Tamura, Endotoxin-induced serum factor that stimulates gamma interferon production. Infect Immun 57, 590-595 (1989).

4. S. Ushio, et al., Cloning of the cDNA for human IFN-gamma-inducing factor, expression in Escherichia coli, and studies on the biologic activities of the protein. J. Immunol. 156, 4274-4279 (1996).

5. T. Ghayur, et al., Caspase-1 processes IFN-gamma-inducing factor and regulates LPS-induced IFNgamma production. Nature 386, 619-623 (1997).

6. Y. Gu, et al., Activation of interferon-gamma inducing factor mediated by interleukin-1beta converting enzyme. Science (80-. ). 275, 206-209 (1997).

7. C. A. Dinarello, Overview of the IL-1 family in innate inflammation and acquired immunity. Immunol. Rev. 281, 8-27 (2018).

8. I. S. Afonina, C. Müller, S. J. Martin, R. Beyaert, Proteolytic Processing of Interleukin-1 Family Cytokines: Variations on a Common Theme. Immunity 42, 991-1004 (2015).

9. R. Medzhitov, et al., MyD88 is an adaptor protein in the hToll/IL-1 receptor family signaling pathways. Mol. Cell 2, 253-258 (1998).

10. H. Wesche, W. J. Henzel, W. Shillinglaw, S. Li, Z. Cao, MyD88: An adapter that recruits IRAK to the IL-1 receptor complex. Immunity 7, 837-847 (1997).

11. Z. Cao, J. Xiong, M. Takeuchi, T. Kurama, D. V. Goeddel, TRAF6 is a signal transducer for interleukin-1. Nature 383, 443-446 (1996).

12. A. Weber, P. Wasiliew, M. Kracht, Interleukin-1ß (IL-1ß) processing pathway. Sci. Signal. 3, cm2$\mathrm{cm} 2$ (2010).

13. C. A. Dinarello, Interleukin-18. Methods 19, 121-132 (1999).

14. C. A. Dinarello, D. Novick, S. Kim, G. Kaplanski, Interleukin-18 and IL-18 binding protein. Front. Immunol. 4, 289 (2013).

15. M. Tone, S. A. Thompson, Y. Tone, P. J. Fairchild, H. Waldmann, Regulation of IL-18 (IFN-gammainducing factor) gene expression. J. Immunol. 159, 6156-6163 (1997).

16. A. Mantovani, C. A. Dinarello, M. Molgora, C. Garlanda, Interleukin-1 and Related Cytokines in the Regulation of Inflammation and Immunity. Immunity 50, 778-795 (2019).

17. D. Novick, et al., Interleukin-18 Binding Protein: A Novel Modulator of the Th1 Cytokine Response. Immunity 10, 127-136 (1999).

18. D. Novick, S. Kim, G. Kaplanski, C. A. Dinarello, Interleukin-18, more than a Th1 cytokine. Semin. Immunol. 25, 439-448 (2013).

19. J. Felix, S. N. Savvides, Mechanisms of immunomodulation by mammalian and viral decoy receptors: insights from structures. Nat. Rev. Immunol. 17, 112-129 (2017).

20. Y. Xiang, B. Moss, IL-18 binding and inhibition of interferon gamma induction by human poxvirusencoded proteins. Proc. Natl. Acad. Sci. U. S. A. 96, 11537-42 (1999).

21. T. L. Born, et al., A Poxvirus Protein That Binds to and Inactivates IL-18, and Inhibits NK Cell Response. J. Immunol. 164, 3246-3254 (2000).

22. B. Krumm, X. Meng, Y. Li, Y. Xiang, J. Deng, Structural basis for antagonism of human interleukin 18 by poxvirus interleukin 18-binding protein. Proc. Natl. Acad. Sci. 105, 20711-20715 (2008).

23. B. Krumm, X. Meng, Z. Wang, Y. Xiang, J. Deng, A unique bivalent binding and inhibition mechanism by the yatapoxvirus interleukin 18 binding protein. PLoS Pathog 8, e1002876 (2012).

24. D. Novick, et al., A novel IL-18BP ELISA shows elevated serum IL-18BP in sepsis and extensive decrease of free IL-18. Cytokine 14, 334-342 (2001).

25. S. Calderara, Y. Xiang, B. Moss, Orthopoxvirus II-18 binding proteins: Affinities and antagonist activities. Virology 279, 22-26 (2001). 
26. N. Tsutsumi, et al., The structural basis for receptor recognition of human interleukin-18. Nat. Commun. 5, 5340 (2014).

27. H. Mühl, et al., Interferon- $\gamma$ Mediates Gene Expression of IL-18 Binding Protein in Nonleukocytic Cells. Biochem. Biophys. Res. Commun. 267, 960-963 (2000).

28. M. Harel, C. Girard-Guyonvarc'h, E. Rodriguez, G. Palmer, C. Gabay, Production of IL-18 Binding Protein by Radiosensitive and Radioresistant Cells in CpG-Induced Macrophage Activation Syndrome. J. Immunol. 205, 1167-1175 (2020).

29. M. Yamamura, et al., Interferon-?-inducing activity of interleukin-18 in the joint with rheumatoid arthritis. Arthritis Rheum. 44, 275-285 (2001).

30. M. Tanaka, et al., Mature form of interleukin 18 is expressed in rheumatoid arthritis synovial tissue and contributes to interferon-gamma production by synovial T cells. J. Rheumatol. $28,1779-1787$ (2001).

31. G. Monteleone, et al., Bioactive IL-18 expression is up-regulated in Crohn's disease. J. Immunol. 163, 143-147 (1999).

32. T. T. Pizarro, et al., IL-18, a novel immunoregulatory cytokine, is up-regulated in Crohn's disease: expression and localization in intestinal mucosal cells. J. Immunol. 162, 6829-6835 (1999).

33. A. Csiszár, G. Nagy, P. Gergely, T. Pozsonyi, E. Pócsik, Increased interferon-gamma (IFN-gamma), IL-10 and decreased IL-4 mRNA expression in peripheral blood mononuclear cells (PBMC) from patients with systemic lupus erythematosus (SLE). Clin. Exp. Immunol. 122, 464-470 (2000).

34. C. K. Wong, E. K. Li, C. Y. Ho, C. W. Lam, Elevation of plasma interleukin-18 concentration is correlated with disease activity in systemic lupus erythematosus. Rheumatol. 39, 1078-1081 (2000).

35. D. Novick, et al., High circulating levels of free interleukin-18 in patients with active SLE in the presence of elevated levels of interleukin-18 binding protein. J. Autoimmun. 34, 121-126 (2010).

36. C. Girard, et al., Elevated serum levels of free interleukin-18 in adult-onset Still's disease. Rheumatol. (United Kingdom) 55, 2237-2247 (2016).

37. J. J. Rodriguez-Smith, et al., Inflammatory biomarkers in COVID-19-associated multisystem inflammatory syndrome in children, Kawasaki disease, and macrophage activation syndrome: a cohort study. Lancet Rheumatol. 3, e574-e584 (2021).

38. C. Lucas, et al., Longitudinal analyses reveal immunological misfiring in severe COVID-19. Nature 584, 463-469 (2020).

39. W. Huang, et al., The Inflammatory Factors Associated with Disease Severity to Predict COVID-19 Progression. J. Immunol. 206, 1597-1608 (2021).

40. H. Flament, et al., Outcome of SARS-CoV-2 infection is linked to MAIT cell activation and cytotoxicity. Nat. Immunol. 22, 322-335 (2021).

41. D. N. Clark, J. L. Markham, C. S. Sloan, B. D. Poole, Cytokine inhibition as a strategy for treating systemic lupus erythematosus. Clin. Immunol. 148, 335-343 (2013).

42. T. Ten Hove, et al., Blockade of endogenous IL-18 ameliorates TNBS-induced colitis by decreasing local TNF-alpha production in mice. Gastroenterology 121, 1372-1379 (2001).

43. C. Gabay, et al., Open-label, multicentre, dose-escalating phase II clinical trial on the safety and efficacy of tadekinig alfa (IL-18BP) in adult-onset Still's disease. Ann. Rheum. Dis. 77, annrheumdis2017-212608 (2018).

44. P. P. Tak, M. Bacchi, M. Bertolino, Pharmacokinetics of IL-18 binding protein in healthy volunteers and subjects with rheumatoid arthritis or plaque psoriasis. Eur. J. Drug Metab. Pharmacokinet. 31, 109-116 (2006).

45. Z. Ma, et al., Augmentation of immune checkpoint cancer immunotherapy with IL18. Clin. Cancer 
604

605

606

607

608

609

610

611

612

613

614

615

616

617

618

619

620

621

622

623

624

625

626

627

628

629

630

631

632

633

634

635

636

637

Res. 22, 2969-2980 (2016).

46. M. J. Robertson, et al., Clinical and biological effects of recombinant human interleukin-18 administered by intravenous infusion to patients with advanced cancer. Clin. Cancer Res. 12, 4265-4273 (2006).

47. A. A. Tarhini, et al., A phase 2, randomized study of SB-485232, rhIL-18, in patients with previously untreated metastatic melanoma. Cancer 115, 859-868 (2009).

48. M. J. Robertson, et al., A dose-escalation study of recombinant human Lnterleukin-18 using two different schedules of administration in patients with cancer. Clin. Cancer Res. 14, 3462-3469 (2008).

49. P. J. Reeves, R. L. Thurmond, H. G. Khorana, Structure and function in rhodopsin: high level expression of a synthetic bovine opsin gene and its mutants in stable mammalian cell lines. Proc. Natl. Acad. Sci. U. S. A. 93, 11487-92 (1996).

50. J. Elegheert, et al., Allosteric competitive inactivation of hematopoietic CSF-1 signaling by the viral decoy receptor BARF1. Nat. Struct. Mol. Biol. 19, 938-47 (2012).

51. J. Felix, et al., Structure and Assembly Mechanism of the Signaling Complex Mediated by Human CSF-1. Structure 23, 1621-31 (2015).

52. Y. Bloch, et al., Structural Activation of Pro-inflammatory Human Cytokine IL-23 by Cognate IL-23 Receptor Enables Recruitment of the Shared Receptor IL-12Rß1. Immunity 48, 45-58.e6 (2018).

53. J. Felix, et al., Structural basis of GM-CSF and IL-2 sequestration by the viral decoy receptor GIF. Nat. Commun. 7, 13228 (2016).

54. K. Fukushima, Y. Ikehara, K. Yamashita, Functional role played by the glycosylphosphatidylinositol anchor glycan of CD48 in interleukin-18-induced interferon- $\gamma$ production. J. Biol. Chem. 280, 18056-18062 (2005).

55. I. J. Tickle, et al., STARANISO (2018).

56. Z. Kato, et al., The structure and binding mode of interleukin-18. Nat. Struct. Biol. 10, 966-971 (2003).

57. Y. Xiang, B. Moss, Determination of the Functional Epitopes of Human Interleukin-18-binding Protein by Site-directed Mutagenesis. J. Biol. Chem. 276, 17380-17386 (2001).

58. S. H. Kim, et al., Structural requirements of six naturally occurring isoforms of the IL-18 binding protein to inhibit IL-18. Proc. Natl. Acad. Sci. U. S. A. 97, 1190-1195 (2000).

59. S. H. Kim, et al., Identification of amino acid residues critical for biological activity in human interleukin-18. J. Biol. Chem. 277, 10998-11003 (2002).

60. G. A, et al., IL-18 mediates sickle cell cardiomyopathy and ventricular arrhythmias. Blood 137, 1208-1218 (2021).

61. L. Au, et al., Cytokine release syndrome in a patient with colorectal cancer after vaccination with BNT162b2. Nat. Med. (2021) https:/doi.org/10.1038/s41591-021-01387-6.

62. R. Karki, et al., Synergism of TNF- $\alpha$ and IFN- $\gamma$ Triggers Inflammatory Cell Death, Tissue Damage, and Mortality in SARS-CoV-2 Infection and Cytokine Shock Syndromes. Cell 184, 149-168.e17 (2021).

63. G. J, et al., Longitudinal proteomic profiling of dialysis patients with COVID-19 reveals markers of severity and predictors of death. Elife 10 (2021).

64. A. Vecchié, et al., IL-18 and infections: Is there a role for targeted therapies? J. Cell. Physiol. 236, 1638-1657 (2021).

65. J. Y, et al., Should we stimulate or suppress immune responses in COVID-19? Cytokine and anticytokine interventions. Autoimmun. Rev. 19 (2020).

66. T. Zhou, et al., IL-18BP is a secreted immune checkpoint and barrier to IL-18 immunotherapy. 
644

645

646

647

648

649

650

651

652

653

654

655

656

657

658

659

660

661

662

663

664

665

666

667

668

669

670

671

672

673

674

675

Nature 583, 609-614 (2020).

67. A. R. Aricescu, W. Lu, E. Y. Jones, A time- and cost-efficient system for high-level protein production in mammalian cells. Acta Crystallogr. D. Biol. Crystallogr. 62, 1243-50 (2006).

68. P. J. Reeves, N. Callewaert, R. Contreras, H. G. Khorana, Structure and function in rhodopsin : Highlevel expression of rhodopsin with restricted and homogeneous $\mathrm{N}$-glycosylation by a tetracyclineHEK293S stable mammalian cell line (2002).

69. T. H. Scheuermann, High-precision, automated integration of multiple isothermal titration calorimetric thermograms: New features of NITPIC. Methods 76, 87-98 (2015).

70. G. Piszczek, SEDPHAT - A platform for global ITC analysis and global multi-method analysis of molecular interactions. Methods 76, 137-148 (2015).

71. F. Madeira, et al., The EMBL-EBI search and sequence analysis tools APIs in 2019. Nucleic Acids Res. 47, W636-W641 (2019).

72. N. Guex, M. C. Peitsch, SWISS-MODEL and the Swiss-Pdb Viewer: An environment for comparative protein modeling. Electrophoresis 18, 2714-2723 (1997).

73. E. J. R. Gupta, S. Brunak, Prediction of N-glycosylation sites in human proteins (2004).

74. C. Steentoft, et al., Precision mapping of the human O-GalNAc glycoproteome through SimpleCell technology. EMBO J. 32, 1478-1488 (2013).

75. Z. Dosztanyi, V. Csizmok, P. Tompa, I. Simon, IUPred: web server for the prediction of intrinsically unstructured regions of proteins based on estimated energy content. Bioinformatics 21, 34333434 (2005).

76. X. Robert, P. Gouet, Deciphering key features in protein structures with the new ENDscript server. Nucleic Acids Res. 42, W320--W324 (2014).

77. W. L. DeLano, The PyMOL Molecular Graphics System. Schrödinger LLC wwwpymolorg Version 1. , http://www.pymol.org (2002).

78. L. Schrödinger, "The PyMOL Molecular Graphics System, Version 1.8" (2015).

79. E. Krissinel, K. Henrick, Inference of Macromolecular Assemblies from Crystalline State. J. Mol. Biol. 372, 774-797 (2007).

80. W. Kabsch, IUCr, XDS. Acta Crystallogr. Sect. D Biol. Crystallogr. 66, 125-132 (2010).

81. A. J. McCoy, et al., Phaser crystallographic software. J. Appl. Crystallogr. 40, 658-674 (2007).

82. P. Emsley, B. Lohkamp, W. G. Scott, K. Cowtan, IUCr, Features and development of Coot. Acta Crystallogr. Sect. D Biol. Crystallogr. 66, 486-501 (2010).

83. P. D. Adams, et al., PHENIX: a comprehensive Python-based system for macromolecular structure solution. Acta Crystallogr. Sect. D Biol. Crystallogr. 66, 213-221 (2010).

84. D. J. Esteban, R. M. L. M. L. Buller, Identification of residues in an orthopoxvirus interleukin-18 binding protein involved in ligand binding and species specificity. Virology 323, 197-207 (2004).

85. S.-H. M. Kim, et al., Site-specific mutations in the mature form of human IL-18 with enhanced biological activity and decreased neutralization by IL-18 binding protein. Proc. Natl. Acad. Sci. 98, 3304-3309 (2002). 
A

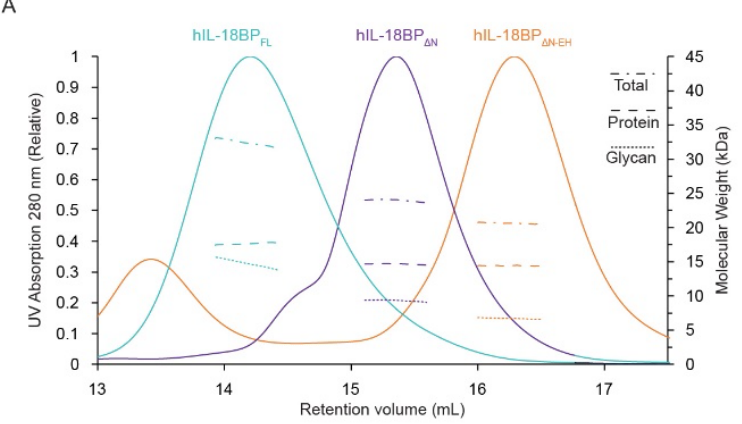

C

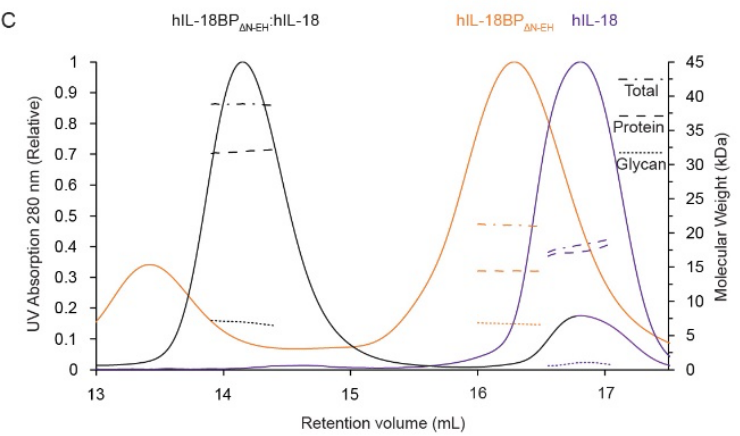

B

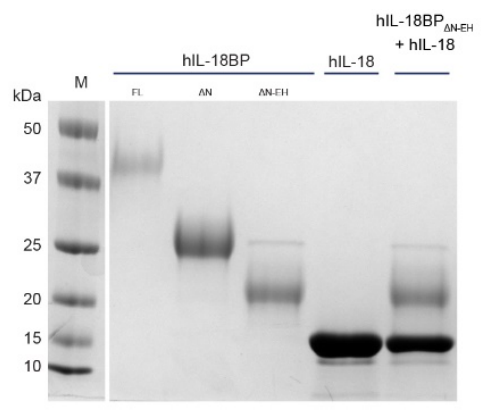

$E$

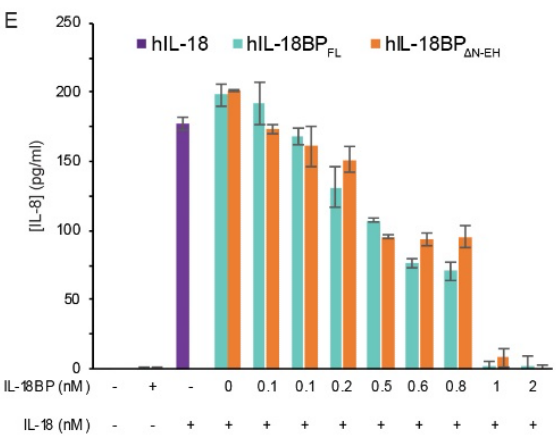

D
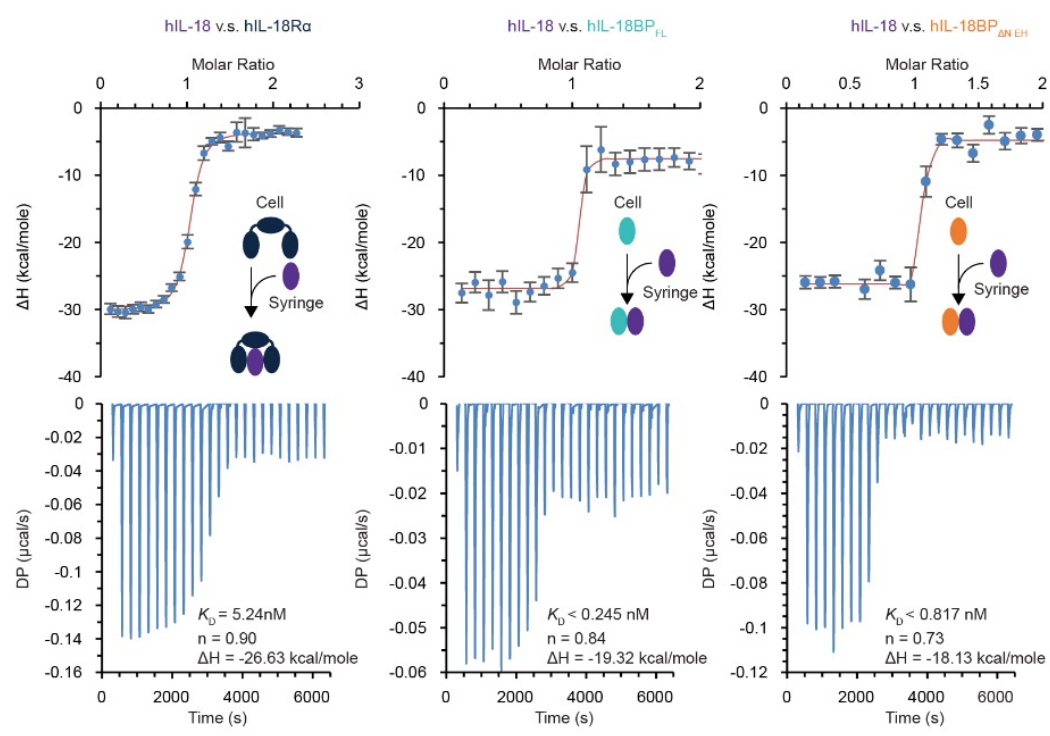

Figure 1. Biochemical characterization and functional activity of human IL-18 and IL-18BP.

(A) SEC-MALLS analysis of hIL-18BP $\mathrm{FL}_{\text {, }}$ hIL-18BP $\triangle \mathrm{N}$ and hIL-18BP $\mathrm{PN}_{\triangle \mathrm{EH}}$ protein (complex) respectively yielding less glycosylated and more homogenous material for crystallization purposes (B) SDS-PAGE of hIL-18BP FL, hIL-18 $8_{\triangle N}$ and hIL-18BP ${ }_{\triangle N-E H}$ show decline in total protein and glycan mass caused by removal of O-glycosylated $\mathrm{N}$-terminus and shaving of $\mathrm{N}$-glycans by Endoglycosidase $\mathrm{H}$ (EH). SDS-PAGE of hIL-18 and hIL-18:hIL-18BP $\triangle \mathrm{AN}$-EH complex show presence and purity of both proteins. 
684 (C) SEC-MALLS analysis of hIL-18:hIL-18BP $\triangle$ N-EH Complex prior to crystallization allow accurate stoichiometry 685 determination (A) \& (C) Full lines show the SEC retention profile of samples $(\sim 0.5-1 \mathrm{mg} / \mathrm{ml})$ detected by 686 UV (left axis, $280 \mathrm{~nm}$ ). Interrupted lines show the molecular weights in kDa of the sample over the course 687 of the SEC peak, split up by protein conjugate analysis in total (dashed and dotted), protein (dashed) and 688 glycan (dotted) mass (right axis).

689 (D) Bar plot of IL-8 levels in conditioned media after hIL-18 stimulation in presence or absence of hIL-18BP FL 690 or IL-18BP $\triangle \mathrm{N}$-EH . Recombinant IL-18 was pre-incubated for $1 \mathrm{~h}$ at $37^{\circ} \mathrm{C}$ with the indicated concentrations of 691 full-length IL-18BP or IL-18BP $\triangle \mathrm{N}$-EH. KG-1 cells were left untreated (UT) or stimulated with IL-18 (purple) (0.5 $692 \mathrm{nM}$ final concentration) +/- IL-18BP (teal and orange), as indicated. After $18 \mathrm{~h}, \mathrm{IL}-8$ concentration in cell 693 culture supernatants was measured by ELISA. Error bars display standard deviation (SEM).

694 (E) Isothermal titration calorimetry (ITC) of hIL-18 in hIL-18Ra, hIL-18BPFL and hIL-18BP $\triangle N-E H$ shows 695 recombinantly produced hIL-18 and hIL-18BP $\triangle \mathrm{N}$-EH are both functionally competent. Bottom plots show the 696 raw baseline-subtracted thermograms while top plots show integrated heats (blue dots) with fitted 697 isotherm (red line). Diagrams in the isotherm plot show which protein was in the cell or syringe and what 698 the expected final complex looks like. 
A

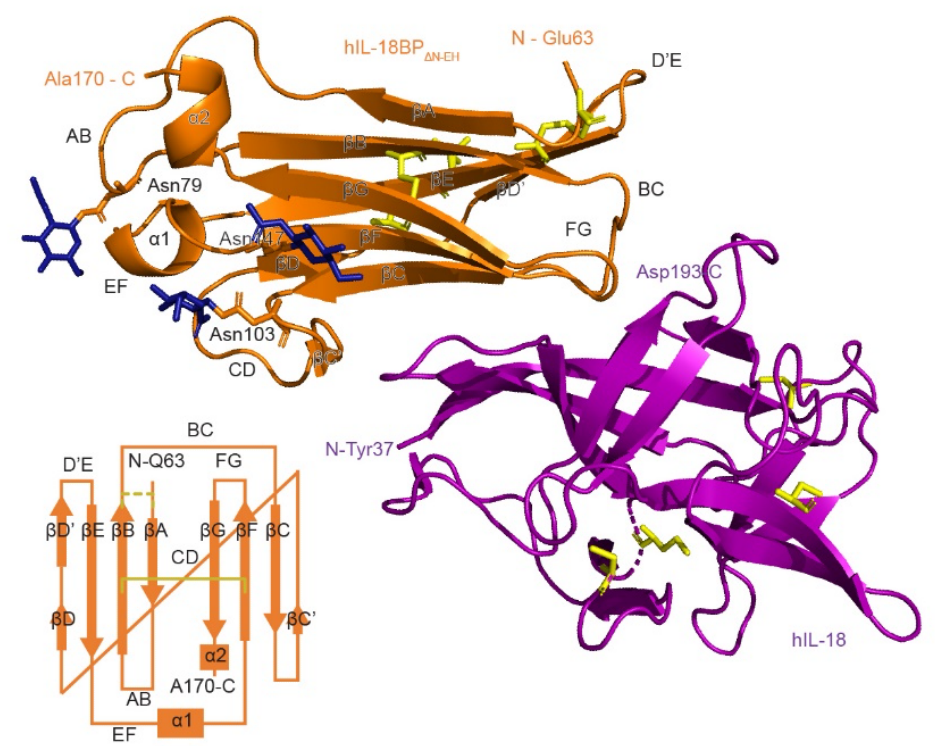

C

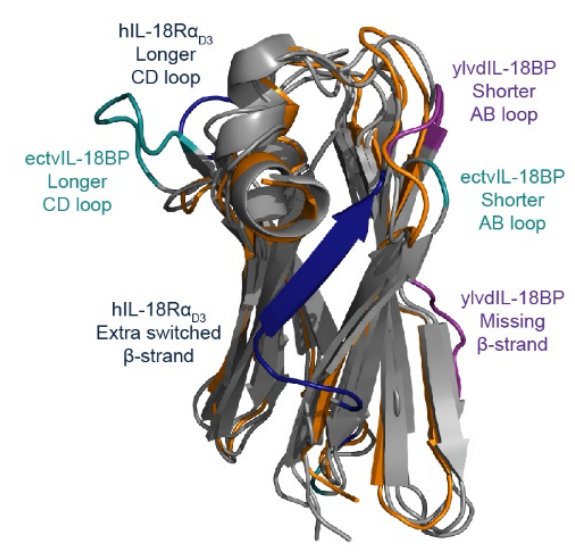

B

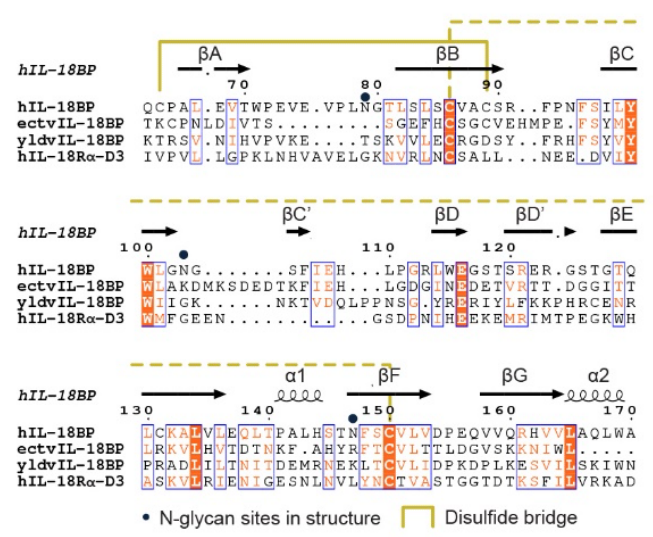

D

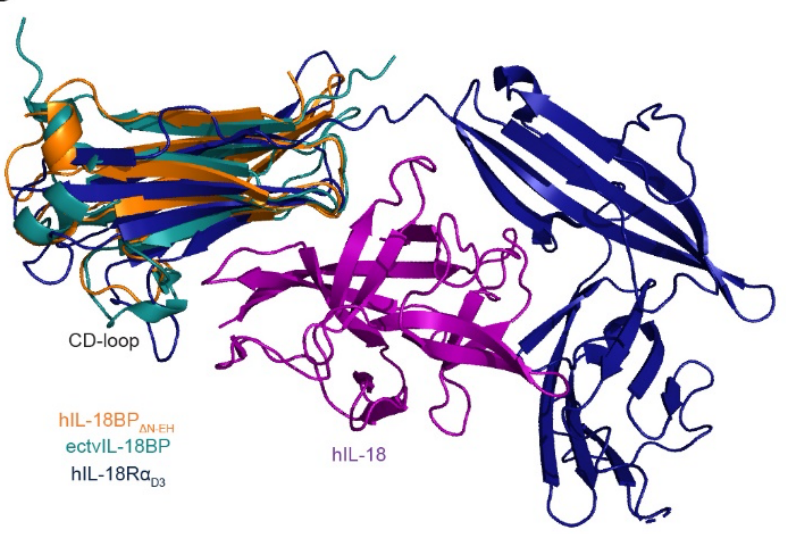

Figure 2. Crystal structure of human IL-18:IL18BP complex and comparisons with human IL-18 receptor and viral decoy receptors.

(A) Cartoon representation of asymmetric unit of the crystal structure of hIL-18:hIL-18BP $\triangle \mathrm{N}-\mathrm{EH}$ displaying hIL-18 (purple) interacting with hIL-18BP ${ }_{\triangle N-E H}$ (orange). Asparagine residues occupied with GlcNAc (stick, blue) are shown in stick representation as well as all cysteines (yellow). Secondary structures of hIL-18BP ${ }_{\triangle N}$ $E H, \beta$-strands, $\alpha$-helices and loops, are labeled in order of occurrence. Additionally, a schematic of the Igfold of hIL-18BP $\triangle \mathrm{N}-\mathrm{EH}$ is shown in the bottom left in which cysteine bridges are represented by yellow lines. (B) Structural sequence alignment based on existing crystal structures performed in Swiss-PdbViewer (72) and visualized by by ESPript (76) shows which residues spatially correspond to each other in the orthologous structures. Secondary structures are annotated by arrows ( $\beta$-strand) and coils ( $\alpha$-helices) above the sequence. Linked disulfides are bridges by yellow lines (full or dashed). Asparagine residues occupied with GlcNAc in the model are labeled with blue dots. Residues with strict identity have an orange background, residues with similarity are colored orange. Groups of high similarity residues are surrounded by a blue box. 
716 (C) Crystal structure overlay of hIL-18BP ${ }_{\triangle N-E H}$ (orange, pdb 7al7), ectvIL-18BP (teal, pdb 3f62(22)), yldvIL717 18BP (purple, pdb 4eee(23)) and hIL-18Ra (dark blue, pdb 3WO4(26)) aligned to hIL-18BP $\triangle$-EH. Only notable 718 differences on the level of secondary structures are colored accordingly, while stretches that align to hIL$71918 \mathrm{BP}_{\triangle \mathrm{N}-\mathrm{EH}}$ are left grey. Structural alignment of these structures shows that the core h-type Ig-fold is 720 maintained with some strand and loop alterations, with an average all atom RMSD of $2.5 \AA$. Compared to 721 human IL-18BP, ectv- and yldvIL-18BP have a shorter AB loop, while ectvIL-18BP has a significantly longer 722 CD loop.

723 (D) Cartoon representation of crystal structures of hIL-18BP $\triangle \mathrm{N}$-EH (orange, pdb 7al7), ectvlL-18BP (teal, pdb 724 3f62(22)), yldvIL-18BP (purple, pdb 4eee(23)) and hIL-18Ra (dark blue, pdb 3WO4(26)) aligned to hIL-18 725 (purple, pdb 7al7). 
bioRxiv preprint doi: https://doi.org/10.1101/2022.02.10.479912; this version posted February 10, 2022. The copyright holder for this preprint (which was not certified by peer review) is the author/funder. All rights reserved. No reuse allowed without permission.

Table 1. Crystallographic data collection and refinement statistics.

728

\begin{tabular}{|c|c|c|}
\hline & Human IL-18:hIL-18BP $\triangle \mathrm{N} E \mathrm{EH}$ & $\begin{array}{c}\text { Human IL-18:hIL-18BP }{ }_{\triangle N} \text { EH } \\
\text { Anisotropy Corrected }\end{array}$ \\
\hline \multicolumn{3}{|l|}{ DATA COLLECTION } \\
\hline Wavelength $(\AA)$ & 0.9762 & 0.9762 \\
\hline Resolution range $(\AA)$ & $59.39-1.80(1.87-1.80)$ & $59.39-1.80(1.85-1.80)$ \\
\hline Space group & C 121 & C 121 \\
\hline Unit cell $\left(a, b, c(\AA), \alpha, \beta, \gamma\left({ }^{\circ}\right)\right)$ & 109.8244 .5260 .289099 .8690 & 109.8244 .5260 .289099 .8690 \\
\hline Total reflections & $183218(17814)$ & $140440(6871)$ \\
\hline Unique reflections & $26507(2546)$ & 20705 (1035) \\
\hline Multiplicity & $6.9(7.0)$ & $6.8(6.6)$ \\
\hline Completeness (\%) & $98.27(96.15)$ & $77.2(49.6)$ \\
\hline Mean I/ $\sigma(I)$ & $15.67(1.49)$ & $18(2.9)$ \\
\hline Wilson B-factor $\left(\AA^{2}\right)$ & 36.35 & n.a. \\
\hline R-meas & $0.053(1.327)$ & $0.048(0.680)$ \\
\hline R-pim & $0.020(0.495)$ & $0.018(0.258)$ \\
\hline $\mathrm{CC} 1 / 2$ & $0.999(0.896)$ & $0.999(0.898)$ \\
\hline $\mathrm{CC}^{*}$ & $1.000(0.972)$ & n.a. \\
\hline \multicolumn{3}{|l|}{ REFINEMENT (59.39 - $1.8 \AA ̊)$} \\
\hline Reflections used in refinement & $26368(2525)$ & $20691(1306)$ \\
\hline Reflections used for R-free & $2640(248)$ & $2062(130)$ \\
\hline R-work/R-free** & $0.207(0.531) / 0.230(0.586)$ & $0.188(0.264) / 0.217(0.320)$ \\
\hline CC(work)/ CC(free) & 0.968 (0.860) / 0.959 (0.812) & 0.874 (0.689) / 0.858 (0.640) \\
\hline Number of non-hydrogen atoms & 2309 & 2309 \\
\hline macromolecules & 2136 & 2136 \\
\hline ligands & 42 & 42 \\
\hline solvent & 131 & 131 \\
\hline RMS bonds $(\AA) /$ angles $\left({ }^{\circ}\right)$ & $0.011 / 1.34$ & $0.011 / 1.34$ \\
\hline Ramachandran favored (\%) & 98.05 & 98.05 \\
\hline Ramachandran allowed (\%) & 1.95 & 1.95 \\
\hline Ramachandran outliers (\%) & 0 & 0 \\
\hline Rotamer outliers (\%) & 0 & 0 \\
\hline Clashscore & 3.48 & 3.48 \\
\hline Average $B$-factor $\left(\AA^{2}\right)$ & 34.68 & 34.68 \\
\hline macromolecules & 34.03 & 34.03 \\
\hline ligands & 66.67 & 66.67 \\
\hline solvent & 35.15 & 35.15 \\
\hline
\end{tabular}

729 Values in parentheses correspond to the highest resolution shell (1.87 - 1.80).

$730 * * 10 \%$ of reflections in $\mathrm{R}$-free set 
bioRxiv preprint doi: https://doi.org/10.1101/2022.02.10.479912; this version posted February 10, 2022. The copyright holder for this preprint (which was not certified by peer review) is the author/funder. All rights reserved. No reuse allowed without permission.
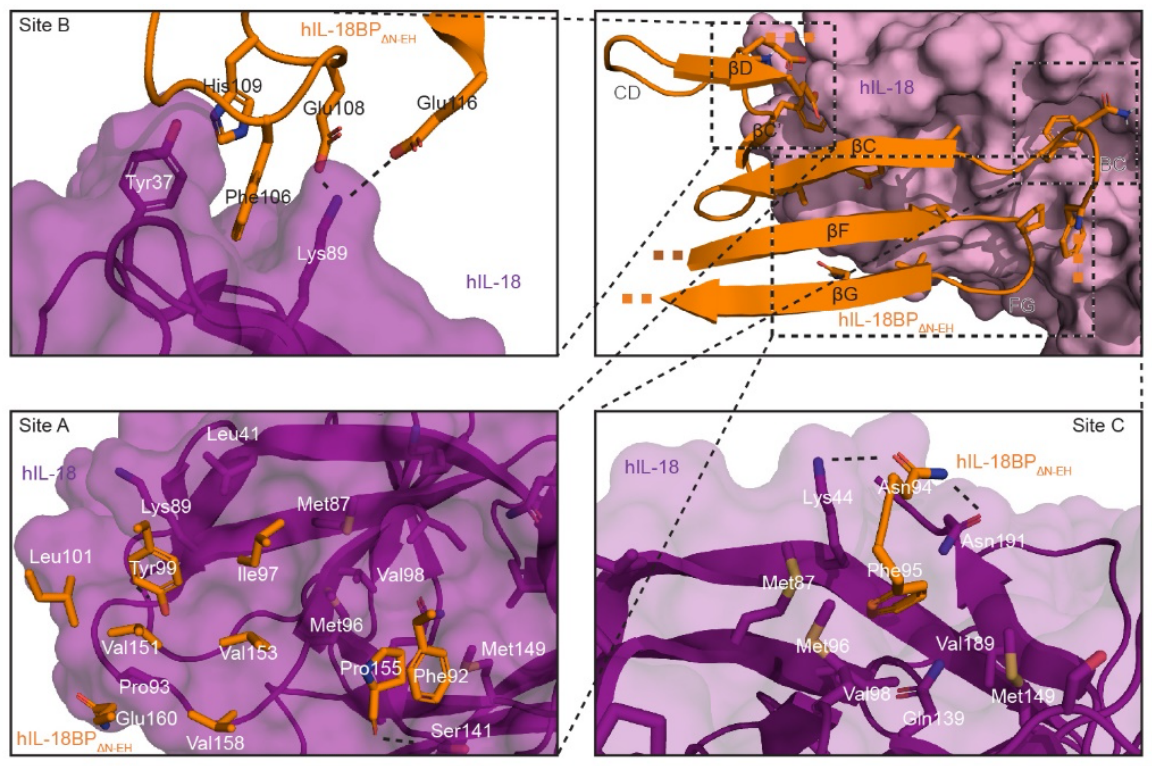

B
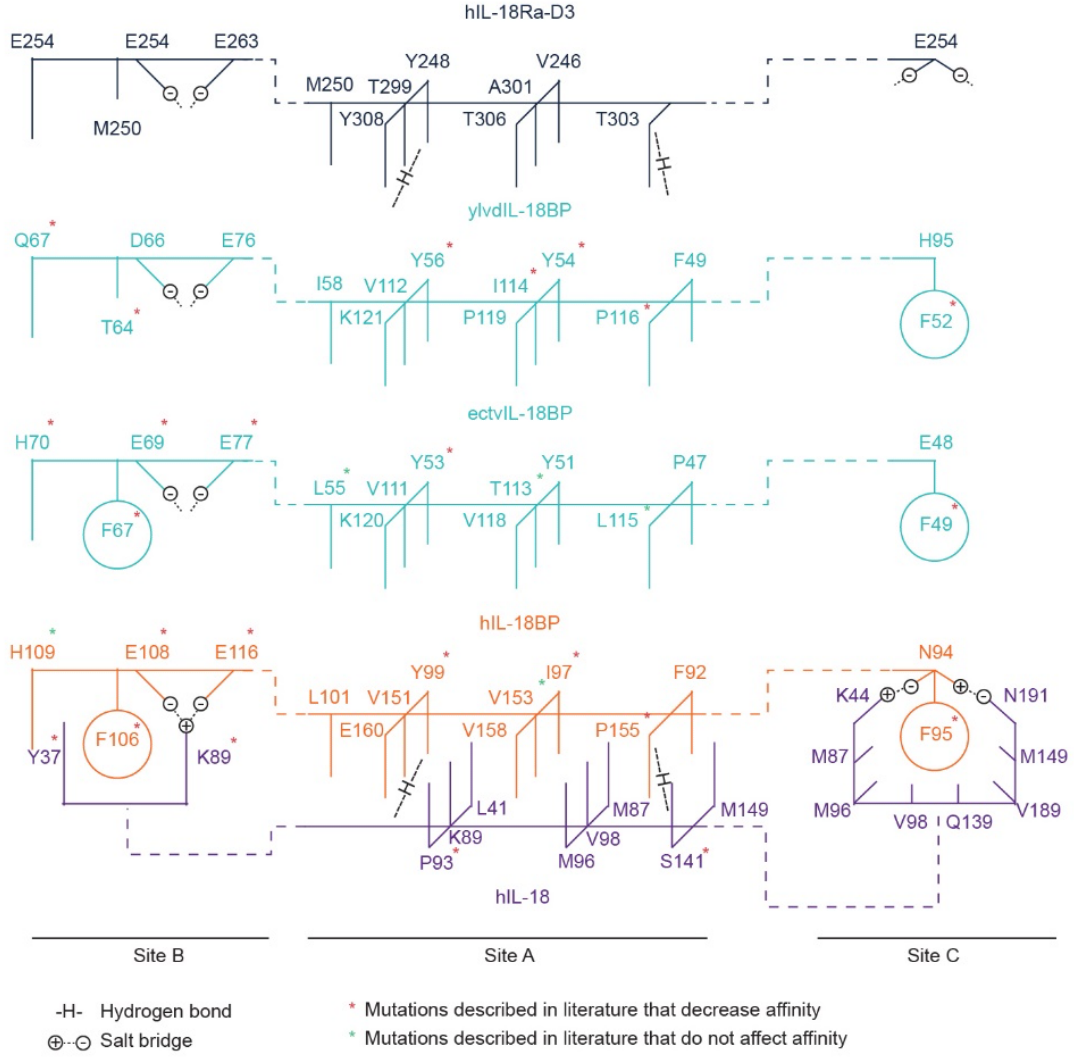
Figure 3. Interaction interface between human IL-18 and human IL-18BP and comparisons with human IL18 receptor and viral receptor decoys

(A) Top right panel shows an overview of interacting structures of hIL-18BP $\Delta \mathrm{N}-\mathrm{EH}$ (cartoon, orange) with

736 hIL-18 (surface, purple). Structures of hIL-18BP $\triangle N-E H$ shown consist out of the bottom half of the beta-

737 sandwich, with the top half and non-interacting loops hidden. Top left, bottom left, and bottom right panels

738 show zoomed-in views of the three main interaction sites $B, A$ and $C$ respectively, with hIL-18BP $\Delta N-E H$ and

739 hIL-18 shown in cartoon representation with a transparent surface overlay for hIL-18. Key residues are

740 shown in stick representation colored by element (blue: nitrogen, red: oxygen, yellow: sulfur, white: polar

741 hydrogens, orange/purple: carbon). Non-polar hydrogens are hidden.

742 (B) Diagrams show simplified interactions at site A, B and C of hIL-18 (purple) with hIL-18BP (orange), viral

743 ectvIL-18BP and ylvdIL-18BP (cyan), and domain 3 of hIL-18Ra (dark blue). The interaction between hIL-18

744 and hIL-18BP consists of 3 sites: A, B and C. Site A consists of interlocking rows of hydrophobic residues.

745 Site B consist of a phenylalanine flanked by hydrophobic side chains supplemented with two salt bridges.

746 Site $\mathrm{C}$ consists of a phenylalanine residing in a large pocket lined by hydrophobic residues supplemented

747 by two salt bridges. Notable differences with ectvIL-18BP and yldvIL-18BP are missing salt bridges at site C,

748 even though a capable side chain is present for ectvIL-18BP. ylvdIL-18BP also has a threonine replacing the

749 phenylalanine at site B. hIL-18Ra-D3 does have the salt bridges at site $\mathrm{C}$, but is lacking the phenylalanine

750 with no substitution, while at site $B$, the phenylalanine is replaced with a methionine. Residues marked with

751 a red asterisk have been reported to have reduced affinity after certain mutations. Residues marked with

752 a green asterisk have been reported to not be affected by certain mutations. Mutagenesis data adopted

753 from previously reported studies(22, 23, 26, 57, 84, 85). 

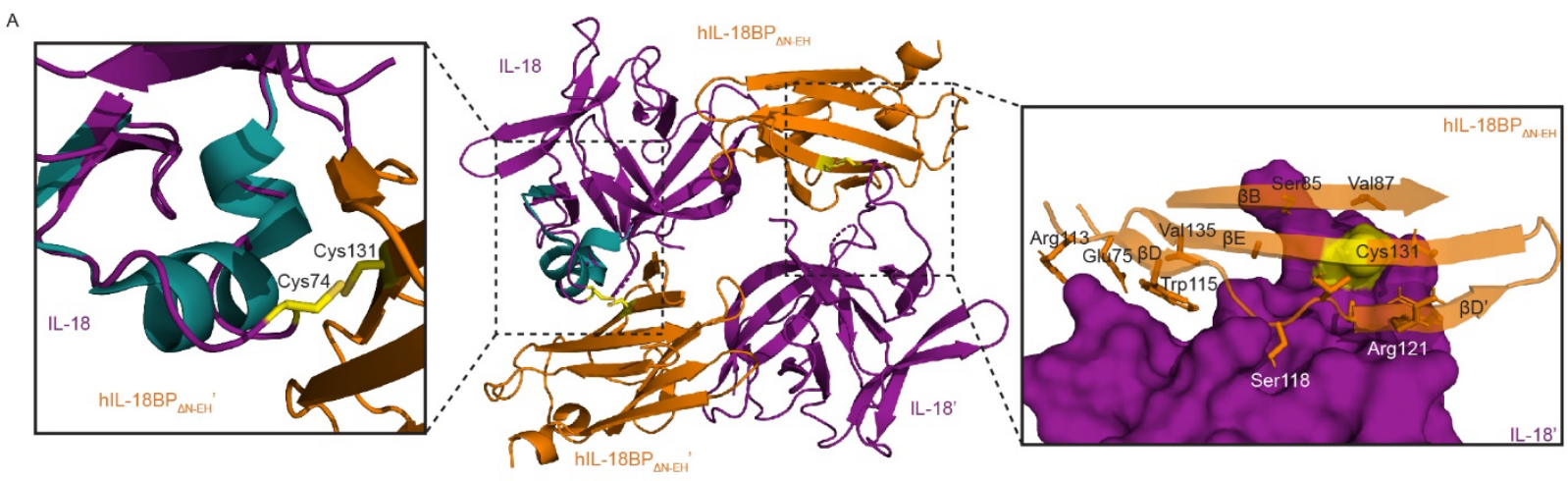

B

c
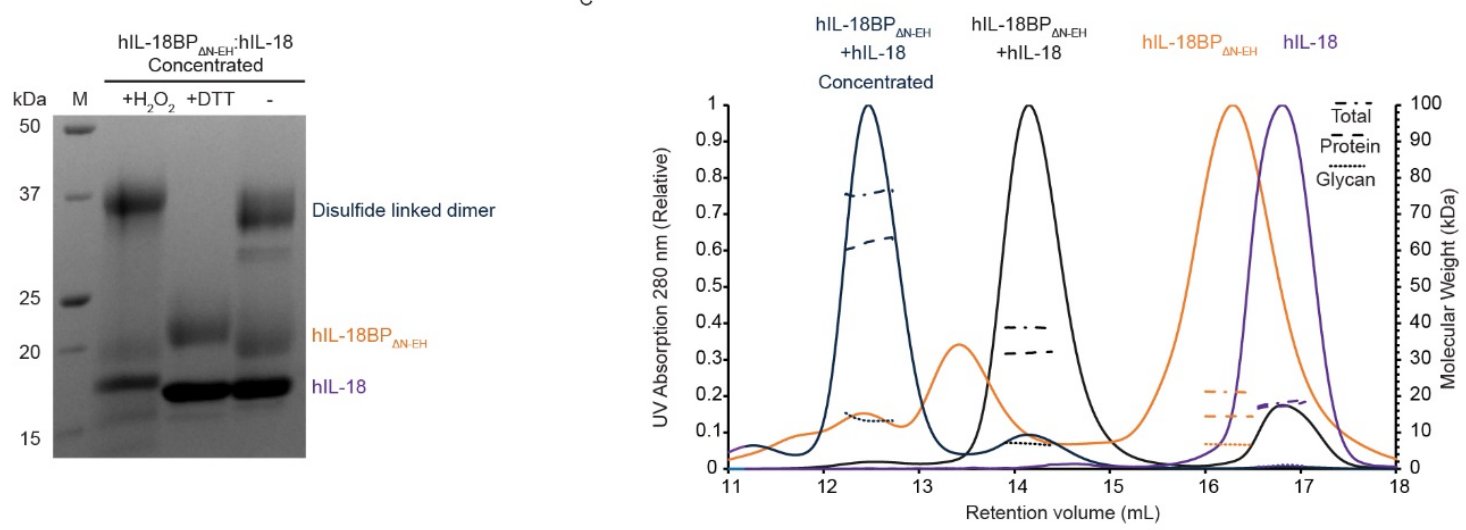

D

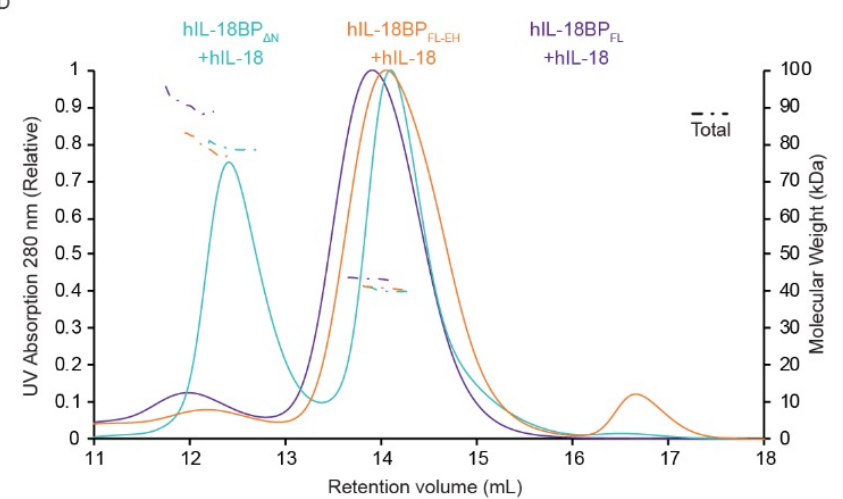

E

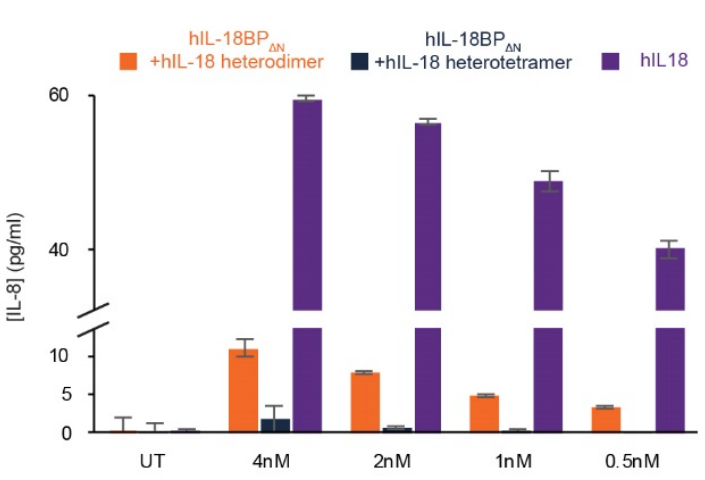

Figure 4. Tetrapartite assembly of human IL-18 and human IL-18BP

(A) Two complexes of hIL-18:hIL-18BP ${ }_{\triangle N}$-EH are linked through an intermolecular disulfide bridge between hIL-18 and hIL-18BP $\triangle \mathrm{N}-\mathrm{EH}$ from neighboring symmetric related unit forming a heterotetrameric assembly with a novel interface. Middle panel shows an overview of the assembly using cartoon representation of hIL-18 (purple or teal) and hIL-18BP $\mathrm{BN}_{\triangle \mathrm{EH}}$ (orange) with the disulfide links involved shown in yellow stick representation. Left panel shows a zoomed-in view with an aligned overlay of the $3_{10} \alpha$-helix from a crystal structure of unbound hIL-18 (teal, pdb 3wo2(26)) showing disruption of the helix upon formation of the disulfide link. The right panel displays the novel interface surrounding the disulfide link with hIL-18 shown in surface representation (purple, pdb 7al7) and hIL-18BP ${ }_{\triangle N-E H}$ in cartoon representation (orange, pdb 7al7) with key residues displayed in stick representation. Strands, loops and key residues are labeled accordingly. 
(B) SDS-PAGE analysis of hIL-18:hIL-18BP $\mathrm{BN}_{\triangle \mathrm{EH}}$ complex sample after concentration to $30 \mathrm{mg} / \mathrm{ml}$ prior to 768 crystallization and diluting back to $0.5 \mathrm{mg} / \mathrm{ml}$ with oxidizing $\left(\mathrm{H}_{2} \mathrm{O}_{2}\right)$, reducing (DTT) or no agents added. 769 Reduction results in dissociation of the otherwise disulfide-linked tetramer.

770 (C) SEC-MALLS analysis of hIL-18, hIL-18BP ${ }_{\triangle N-E H}$ and hIL-18:hIL-18BP $\triangle$ N-EH complex before and after 771 concentration to $30 \mathrm{mg} / \mathrm{ml}$ prior to and diluting back to $0.5 \mathrm{mg} / \mathrm{ml}$. Line plots show the SEC retention profile 772 of samples detected by UV (left axis). Interrupted lines plot the molecular weights of the sample over the 773 course of the SEC peak, dissected by protein conjugate analysis in total (dashed and dotted), protein 774 (dashed) and glycan (dotted) mass (right axis).

775 (D) SEC-MALLS analysis of hIL-18:hIL-18BP $\triangle \mathrm{P}_{\Delta \mathrm{N}}$ and hIL-18:hIL-18BP $\mathrm{FL}_{\mathrm{F}}$ complex sample after concentration to $77630 \mathrm{mg} / \mathrm{ml}$ and diluting back to $0.5 \mathrm{mg} / \mathrm{ml}$. Line plots show the SEC retention profile of samples detected by 777 UV (left axis). Interrupted lines plot the molecular weights of the sample over the course of the SEC peak, 778 dissected by protein conjugate analysis in total (dashed and dotted), protein (dashed) and glycan (dotted) 779 mass (right axis).

780 (E) Bar plot of IL-8 levels ( $\mathrm{y}$-axis, $\mathrm{pg} / \mathrm{mL}$ ) in conditioned media after human IL-18 stimulation in presence or 781 absence of dimeric or tetrameric IL-18BP ${ }_{\triangle N-E H}: I L-18$ complex. Recombinant IL-18 was pre-incubated for $1 \mathrm{~h}$ 782 at $37^{\circ} \mathrm{C}$ with the indicated concentrations of full-length IL-18BP or IL-18BP $\triangle \mathrm{N}$-EH. KG-1 cells were left 783 untreated (UT) or stimulated with IL-18 (purple) (0.5 nM final concentration) +/- IL-18BP (teal and orange), 784 as indicated. After $18 \mathrm{~h}, \mathrm{IL}-8$ concentration in cell culture supernatants was measured by ELISA. Error bars 785 display standard deviation (SEM). 


\title{
Supplementary Information for
}

\section{Structural basis of human IL-18 sequestration by the decoy receptor IL-18 binding protein (IL-18BP) in inflammation and tumor immunity}

Sammy Detry ${ }^{1,2, \#, ~ J u l i e ~ A n d r i e s ~}{ }^{1,2, \#}$, Yehudi Bloch ${ }^{1,2}$, Cem Gabay $^{3}$, Danielle Clancy ${ }^{1,2}$, Savvas N. Savvides ${ }^{1,2, *}$

\author{
1 Unit for Structural Biology, Department of Biochemistry and Microbiology, Ghent University, Technologiepark 71, 9052 \\ Ghent, Belgium. \\ 2Unit for Structural Biology, VIB Center for Inflammation Research, Technologiepark 71, 9052 Ghent, Belgium. \\ ${ }^{3}$ Division of Rheumatology, Department of Medicine, Geneva University Hospitals \& Faculty of Medicine University of \\ Geneva, CH-1211 Geneva 14, Switzerland. \\ \#These authors contributed equally \\ *Corresponding author: savvas.savvides@ugent.be
}

\section{This PDF file includes:}

Figures S1 to S2

Tables

SI References 


\section{SUPPLEMENTAL FIGURE 1 (legend below)}

A
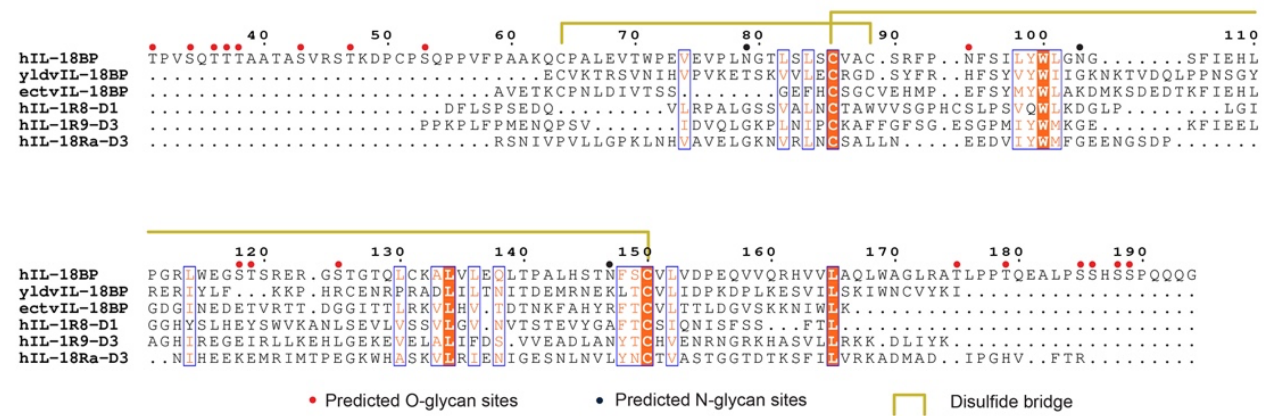

B

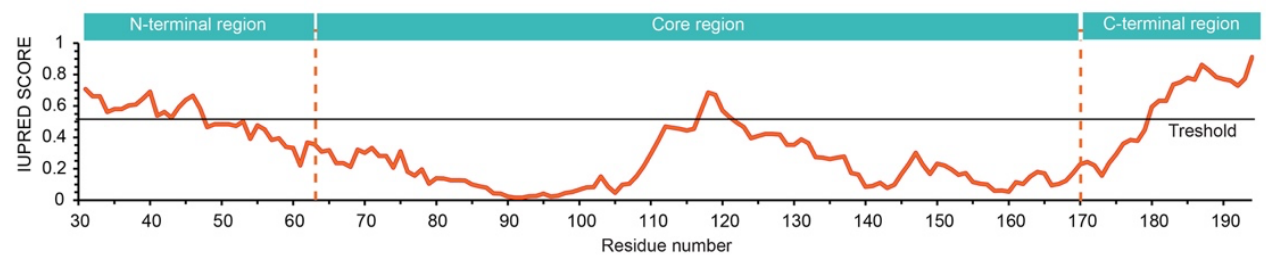

Supplemental Figure 1. Sequence analysis for protein construct optimization

(A) Sequence alignment performed by Clustal Omega ${ }^{1}$ visualized by ESPript ${ }^{2}$ of hIL-18BP and related sequences. The core-region was delineated based on the sequence alignment, disordered region prediction and existing crystal structures of viral IL-18BPs. Gaps in the sequence alignment are represented by dots. Residues with strict identity have an orange background, residues with similarity are colored orange. Groups of high similarity residues are surrounded by a blue box. Predicted $\mathrm{N}^{3}$ and $\mathrm{O}-$ glycan ${ }^{4}$ sites are displayed as blue and red dots respectively. Linked disulfides are represented by yellow lines (full or dashed).

(B) IUPRED prediction ${ }^{5}$ of disordered regions in human IL-18BP show that the extended $\mathrm{N}$ - and Ctermini are likely disordered and flexible. Disorder threshold is represented by a black horizontal line. Delineation of tested terminal truncations is represented by vertical dashed orange lines. 


\section{SUPPLEMENTAL FIGURE 2 (legend below)}
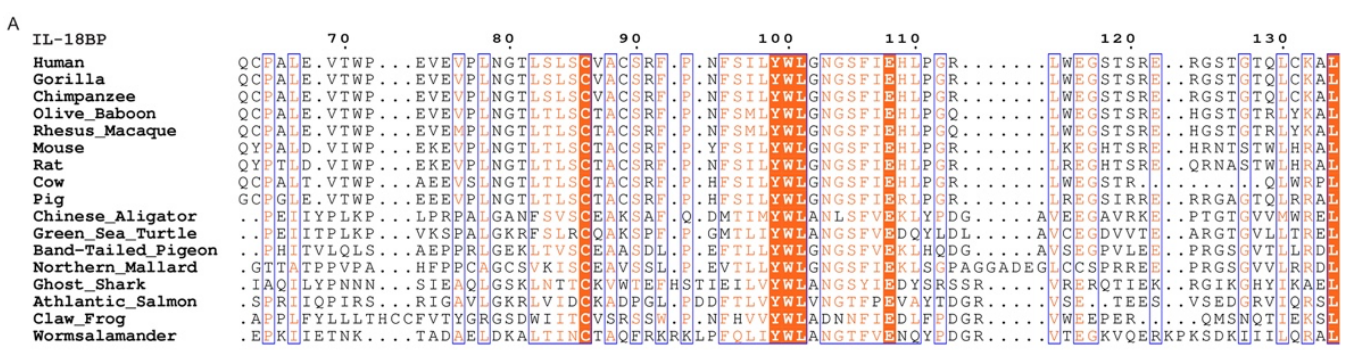

\section{IL-18BP}

Human
Gorilla

Chimpanzee

Olive_Baboon

Mouse
Rat

Cow

Chinese_Aligator

Green_Sea_Turtie

Band-Tailed_Pigeon
Northern_Mallard

Ghost_Shark

Athlantic_.

Claw_Frog
Wormsalamander
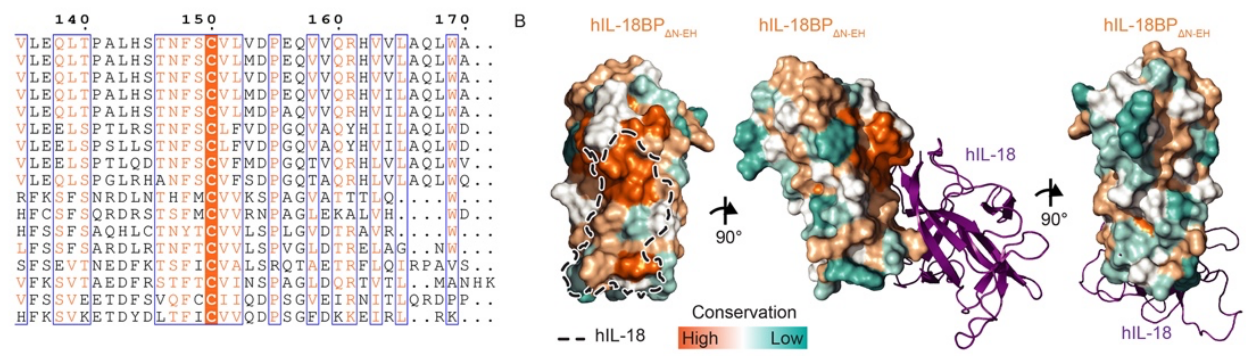

Supplemental Figure 2. Sequence conservation for hIL-18BP

(A) Sequence alignment of core region of orthologous IL-18BPs between species across the animal kingdom. Residues with strict identity have an orange background, residues with similarity are colored orange. Groups of high similarity residues are surrounded by a blue box. Color scale above the sequence of hIL-18BP represents conservation levels determined by ConSurf ${ }^{6}$, with high conservation colored in orange, low conservation levels in teal and white in between. The same scale is used in panel $\mathrm{B}$.

(B) Surface representation of hIL-18BP ${ }_{\triangle N}$-EH colored by residue conservation. hIL-18 binding to hIL$18 \mathrm{BP}_{\triangle \mathrm{N}-\mathrm{EH}}$ is either displayed in cartoon representation (purple, two right panels) or shown as a black dashed line representing the patch it covers on hIL-18BP $P_{\triangle N-E H}$. 
Supplementary Table 1. Molecular weights and binding parameters of recombinant hIL-18 and hIL18BP proteins

\begin{tabular}{|c|c|c|c|c|c|c|}
\hline \multirow[b]{2}{*}{$\begin{array}{l}\text { Protein } \\
\text { (complex) }\end{array}$} & \multirow[b]{2}{*}{$\begin{array}{l}\text { MW } \\
(\mathrm{kDa})\end{array}$} & \multicolumn{3}{|c|}{$\begin{array}{c}\text { Molecular Weight } \\
\text { determined by MALLS }\end{array}$} & \multicolumn{2}{|c|}{$\begin{array}{l}\text { Binding to hIL-18 } \\
\text { determined by ITC }\end{array}$} \\
\hline & & $\begin{array}{c}\text { Total MW } \\
\pm \mathrm{SD} \\
(\mathrm{kDa})\end{array}$ & $\begin{array}{c}\text { Protein MW } \\
\pm \mathrm{SD} \\
(\mathrm{kDa})\end{array}$ & $\begin{array}{c}\text { glycan MW } \\
\pm \mathrm{SD} \\
(\mathrm{kDa})\end{array}$ & $\begin{array}{c}K_{\mathrm{D}} \\
(95 \% \mathrm{Cl}) \\
(\mathrm{nM})\end{array}$ & $\begin{array}{c}\Delta \mathrm{H} \\
(95 \% \mathrm{Cl}) \\
(\mathrm{kcal} / \mathrm{mole})\end{array}$ \\
\hline hIL-18 & 18.2 & $18.2 \pm 0.5$ & - & - & - & - \\
\hline hIL-18BP FL & 17.6 & $32.4 \pm 0.5$ & $17.6 \pm 0.1$ & $14.8 \pm 0.6$ & $\begin{array}{c}\text { n.d. } \\
(-\infty-0.245)\end{array}$ & $\begin{array}{c}-19.32 \\
(-18.33- \\
20.32)\end{array}$ \\
\hline hIL-18BP ${ }_{\Delta N}$ & 14.4 & $24.0 \pm 0.2$ & $14.65 \pm 0.06$ & $9.3 \pm 0.1$ & - & - \\
\hline hIL-18BP ${ }_{\triangle N-E H}$ & 14.4 & $21.12 \pm 0.09$ & $14.42 \pm 0.02$ & $6.71 \pm 0.08$ & $\begin{array}{c}\text { n.d. } \\
(-\infty-0.817)\end{array}$ & $\begin{array}{c}-18.13 \\
(-16.91--19.36)\end{array}$ \\
\hline $\begin{array}{c}\text { hIL-18 } \\
\text { hIL-18BP }{ }_{\triangle N-E H}\end{array}$ & 32.6 & $38.80 \pm 0.06$ & $31.9 \pm 0.2$ & $6.9 \pm 0.2$ & 1 & 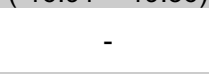 \\
\hline hIL-18Ra & 35.4 & - & - & - & $\begin{array}{c}5.24 \\
(7.94-3.38)\end{array}$ & $\begin{array}{c}-26.63 \\
(-25.59- \\
27.77)\end{array}$ \\
\hline
\end{tabular}

MW: Molecular weight, SD: Standard Deviation, Cl: Confidence interval, MALLS: Multi-Angle Laser Light Scattering, ITC: Isothermal Titration Calorimetry 


\section{SI References}

1. Madeira, F. et al. The EMBL-EBI search and sequence analysis tools APIs in 2019. Nucleic Acids Res. 47, W636-W641 (2019).

2. Robert, X. \& Gouet, P. Deciphering key features in protein structures with the new ENDscript server. Nucleic Acids Res. 42, W320--W324 (2014).

3. R. Gupta, E. J. \& Brunak, S. Prediction of N-glycosylation sites in human proteins. (2004).

4. Steentoft, C. et al. Precision mapping of the human O-GalNAc glycoproteome through SimpleCell technology. EMBO J. 32, 1478-1488 (2013).

5. Dosztanyi, Z., Csizmok, V., Tompa, P. \& Simon, I. IUPred: web server for the prediction of intrinsically unstructured regions of proteins based on estimated energy content. Bioinformatics 21, 3433-3434 (2005).

6. Ashkenazy, H. et al. ConSurf 2016: an improved methodology to estimate and visualize evolutionary conservation in macromolecules. Nucleic Acids Res. 44, W344--W350 (2016). 